\title{
GALOIS $p$-GROUPS AND GALOIS MODULES
}

\author{
SUNIL CHEBOLU, JÁN MINÁČ AND ANDREW SCHULTZ \\ Dedicated to Professor Albrecht Pfister
}

\begin{abstract}
The smallest non-abelian $p$-groups play a fundamental role in the theory of Galois $p$-extensions. We illustrate this by highlighting their role in the definition of the norm residue map in Galois cohomology. We then determine how often these groups-as well as other closely related, larger $p$-groups-occur as Galois groups over given base fields. We show further how the appearance of some Galois groups forces the appearance of other Galois groups in an interesting way.
\end{abstract}

1. Introduction. From its beginning, Galois theory has carried an aura of mystery and depth. Despite remarkable progress, some very basic problems remain open. Given a base field $F$ and a finite group $G$, the inverse Galois problem asks whether there is a Galois extension $K / F$ with Galois group $G$. When $F$ is the field of rational numbers we do not have a solution for all $G$. However, in the remarkable paper [46], Shafarevich showed that each solvable group $G$ appears as a Galois group over any algebraic number field. (See also [47] for corrections related to problems caused by the prime 2.) For a comprehensive treatment of Shafarevich's theorem over any global field, see [39, subsection 9.6]. For another nice exposition of Shafarevich's theorem in the original case of algebraic number fields, see [18, Chapter V, Section $6]$. For a special case of solvable groups $G$ whose order is a power of a prime $p$, see [48].

2010 AMS Mathematics subject classification. Primary 12F10, Secondary $12 \mathrm{~F} 12$.

Keywords and phrases. Galois groups, p-groups, Galois modules, enumerating Galois extensions, norm residue isomorphism.

The first author is partially supported by NSA grant No. H98230-13-1-0238. The second author is partially supported by NSERC grant No. R0370A01.

Received by the editors on July 14, 2014, and in revised form on October 28, 2014. 
Although in the above works there are some considerable technical issues, some basic principles can be explained briefly. The first basic ingredient is the description of all Galois extensions $K / F$ with Galois group $\mathbb{Z} / p$ over any field $F$. If $F$ contains a primitive $p$ th root of unity, this problem has a classical, elegant solution described by Kummer theory; if $\operatorname{char}(\mathrm{F})=p$, the problem is solved by Artin-Schreier theory, and when $\operatorname{char}(\mathrm{F}) \neq p$ and $F$ does not contain the primitive $p$ th root of unity, one can use Galois descent (see [23, Chapter 6]). The appearance of elementary $p$-abelian groups can be described in this language as well. Indeed, for any field $F$, there exists an $\mathbb{F}_{p}$-space $J(F)$ so that subspaces of $J(F)$ of dimension $k$ are in correspondence with elementary $p$-abelian extensions $L / F$ of rank $k$. (We will consider $J(F)$ more fully in Section 3.)

In order to build a given group $G$ of prime-power order as a Galois group over a given field $F$, it is natural to consider Galois embedding problems. Consider the short exact sequence:

$$
1 \longrightarrow \operatorname{Gal}(L / K) \longrightarrow \operatorname{Gal}(L / F) \longrightarrow \operatorname{Gal}(K / F) \longrightarrow 1
$$

from Galois theory. If, given $G \rightarrow Q$ and $\operatorname{Gal}(K / F) \simeq Q$, can a Galois extension $L / F$ as above be found so that $\operatorname{Gal}(L / F) \simeq G$, with the natural map from Galois theory corresponding to the original surjection $G \rightarrow Q$ ? This is the Galois embedding problem for $G \rightarrow Q$ over the extension $K / F$.

First consider $p>2$. The smallest nonabelian groups of primepower order have order $p^{3}$, and, up to group isomorphism, there are exactly two such groups (see, e.g., [8, pages 185-186]). The Heisenberg group, which we write $H_{p^{3}}$, is the unique nonabelian group of order $p^{3}$ and exponent $p$. This group is isomorphic to $U_{3}\left(\mathbb{F}_{p}\right)$, the group of upper triangular matrices over a field with $p$-elements and all diagonal elements 1. The modular group, which we write $M_{p^{3}}$, is the unique nonabelian group of order $p^{3}$ and exponent $p^{2}$. If $p=2$, there are also two nonabelian groups of order 8: the dihedral group $D_{4}$ (isomorphic to $\left.U_{3}\left(\mathbb{F}_{2}\right)\right)$ and the quaternion group $Q_{8} . H_{p^{3}}$ and $M_{p^{3}}$ have the following presentations by generators and relations:

$$
\begin{aligned}
& H_{p^{3}}=\left\langle\sigma, \tau, \omega \mid \sigma^{p}=\tau^{p}=\omega^{p}=[\omega, \sigma]=[\omega, \tau]=\mathrm{id},[\sigma, \tau]=\omega\right\rangle \\
& M_{p^{3}}=\left\langle x, y \mid y^{p^{2}}=x^{p}=\mathrm{id},[x, y]=y^{p}\right\rangle=\langle y\rangle \rtimes\langle x\rangle .
\end{aligned}
$$


It is worth observing that $M_{p^{3}}$ is one group in the larger family of groups of the form

$$
M_{p^{n}}=\left\langle y: y^{p^{n-1}}=1\right\rangle \rtimes\left\langle x: x^{p}=1\right\rangle .
$$

When $p=2$ and $n>3$, there are four non-abelian groups of order $2^{n}$ which have an element of order $2^{n-1} ; M_{2^{n}}$ is obviously one of them, and some automatic realization results for these four groups were considered in [19]. (Jensen uses $F_{2^{n}}$ to denote the group we are calling $M_{2^{n}}$.) For odd $p$, the group $M_{p^{n}}$ is the only non-abelian group of order $p^{n}$ which contains an element of order $p^{n-1}$ (see, e.g., [16, subsection 12.5]). Michailov [27] described the realizability conditions and Galois extensions for $M_{p^{n}}$, as well as several other groups related to this group.

The nonabelian groups of order $p^{3}$, along with cyclic groups of order dividing $p^{2}$, play a surprising basic role in the structure of some canonical quotients of absolute Galois groups of fields containing a primitive $p$ th root of unity. For $p=2$, based on the work of Villegas [33], it was shown that the fixed field of the third term of the 2-descending central sequence of the absolute Galois group is the compositum of all Galois extensions of the base field with Galois group isomorphic to $\mathbb{Z} / 2, \mathbb{Z} / 4$ or the dihedral group of order 8 . Similar results were obtained [11] for $p>2$ with groups $\mathbb{Z} / p, \mathbb{Z} / p^{2}$ and $M_{p^{3}}$. When replacing the 2-descending central sequence by the Zassenhaus descending sequence [12], an analogous result for groups $\mathbb{Z} / p$ and $H_{p^{3}}$ was shown. On the other hand, it was shown $[7,12,13,34]$ that these quotients of absolute Galois groups of fields as above encode crucial information about Galois cohomology, valuations and orderings of fields. These fundamental results can also be viewed as precursors to current investigations $[\mathbf{9}, \mathbf{1 0}, \mathbf{1 7}, \mathbf{3 0}, \mathbf{3 6}, \mathbf{3 7}, \mathbf{3 8}]$ related to Massey products in Galois cohomology.

If $F$ is a field containing a primitive $p$ th root of unity $\xi_{p}$, and if $a, b \in F^{\times}$give rise to a $\mathbb{Z} / p \times \mathbb{Z} / p$ extension $F(\sqrt[p]{a}, \sqrt[p]{b}) / F$, then it is known (see [24, (3.6.4)], [26, Theorem 3 (A)], [28, Theorem 3.1]) that the embedding problem corresponding to

$$
1 \longrightarrow \mathbb{Z} / p \longrightarrow H_{p^{3}} \longrightarrow \mathbb{Z} / p \times \mathbb{Z} / p \longrightarrow 1
$$

is solvable over $F(\sqrt[p]{a}, \sqrt[p]{b})$ if and only if $b \in N_{F(\sqrt[p]{a}) / F}\left(F(\sqrt[p]{a})^{\times}\right)$. 
The analogous embedding problem for $M_{p^{3}}$ is solvable over $F(\sqrt[p]{a}, \sqrt[p]{b})$ if and only if

$$
b \xi_{p}^{k} \in N_{F(\sqrt[p]{a}) / F}\left(F(\sqrt[p]{a})^{\times}\right) \quad \text { for some } k \in \mathbb{Z} \backslash p \mathbb{Z}
$$

(see [6, Theorem 1], [26, pages 523-524, Corollary], [28, Theorem 3.2]). It is also known that the existence of an extension $K / F$ with $\operatorname{Gal}(K / F) \simeq H_{p^{3}}$ implies the existence of an extension $L / F$ with $\operatorname{Gal}(L / F) \simeq M_{p^{3}}($ see $[6$, Theorem 2$])$.

In some special cases, more precise results were obtained. Letting $\nu(F, G)$ be the number of extensions of $F$ whose Galois group is $G$ (in a fixed algebraic closure $\bar{F})$, Brattström has proved ([6, Theorem 5]) that, when $\operatorname{char}(\mathrm{F})=p$ or $\xi_{p^{2}} \in F$, one has

$$
\nu\left(F, M_{p^{3}}\right)=\left(p^{2}-1\right) \nu\left(F, H_{p^{3}}\right) .
$$

The purpose of this paper is to investigate $H_{p^{3}}, M_{p^{3}}$ and their closely related $p$-groups as Galois groups. We investigate the number of Galois extensions with given Galois group $G$ as above and the interrelation between these numbers. Section 2 discusses the fundamental relation in Galois cohomology. Assume that $a$ and $1-a$ are non-zero elements in a field $F$, and $\xi_{p} \in F$. Then, by Kummer theory, we have associated classes $(a),(1-a) \in H^{1}\left(G_{F}, \mathbb{F}_{p}\right)$. Here $G_{F}$ is the absolute Galois group of $F$ and $H^{i}\left(G_{F}, \mathbb{F}_{p}\right)$ is the $i$ th group cohomology of $G_{F}$ with the $\mathbb{F}_{p}$-coefficients viewed as a trivial module over $G_{F}$. Then $(a) \cup(1-a)=0 \in H^{2}\left(G_{F}, \mathbb{F}_{p}\right)$, a result known as the Bass-Tate lemma (see Proposition 2.1 below). We discuss how this relation is connected to the solvability of embedding problem (1.2) over the field $F(\sqrt[p]{a}, \sqrt[p]{1-a})$. We will use this as motivation for providing an "elementary" proof of this vanishing for $p>2$ akin to the result of Pfister [40] for $p=2$. Pfisters' proof is remarkable as it uses nothing but the definition of Galois cohomology, but the impetus behind the selection of the desired coboundary is unexplained. In our treatment, we also keep the elementary nature of the proof, but we shed light on the construction of the desired coboundary.

The Bass-Tate lemma has a fairly short proof (see Section 2), but it is nevertheless a deep statement which implies automatic realizations for Galois groups $H_{p^{3}}, \mathbb{Z} / 4$ and the dihedral group of order 8 . It also directly connects Galois theory to the crucial relation between addition and multiplication in the base field. This connection has 
some profound consequences for birational anabelian geometry (see $[3,4,5,43,44,50,51])$.

In Section 3, we change our methodology for investigating $H_{p^{3}}$ and $M_{p^{3}}$ extensions by replacing the embedding problem from (1.2) to one that comes from a short exact sequence of the form:

$$
1 \longrightarrow \mathbb{Z} / p \times \mathbb{Z} / p \longrightarrow G \longrightarrow \mathbb{Z} / p \longrightarrow 1 \text {. }
$$

(Both $H_{p^{3}}$ and $M_{p^{3}}$ fit in such an exact sequence.) If $K / F$ is the base extension satisfying $\operatorname{Gal}(K / F) \simeq \mathbb{Z} / p$, this new perspective allows us to parameterize solutions to these embedding problems over $K / F$ by certain $\operatorname{Gal}(K / F)$-submodules within $J(K)$. This change in perspective also allows us to exhibit $H_{p^{3}}$ and $M_{p^{3}}$ within a larger family of $p$-groups (namely, those which can be written as an extension of $\mathbb{Z} / p^{n}$ by a cyclic $\mathbb{F}_{p}\left[\mathbb{Z} / p^{n}\right]$-module) for which the solvability of the associated embedding problem is again tied to the appearance of certain Galois modules in $J(K)$. In the final two sections, we use this module-theoretic perspective to give generalizations of some of the known results concerning the appearance of $M_{p^{3}}$ and $H_{p^{3}}$ as Galois groups to this broader family of groups.

2. Norm residue homomorphism and Galois modules. One of the most exciting theorems from the last decade was the proof of the norm residue isomorphism (previously, the Bloch-Kato conjecture). Before stating this theorem, we remind the reader of some important terms. Throughout this section, we assume that $p$ is a given prime number and that $F$ is a field which contains a primitive $p$ th root of unity $\xi_{p}$. We denote the separable closure of $F$ by $F_{\text {sep }}$ and the associated absolute Galois group by $G_{F}:=\operatorname{Gal}\left(\bar{F}_{\text {sep }} / F\right)$. When we speak of the cohomology of $F$, we mean the cohomology groups associated to the trivial $G_{F}$-module $\mathbb{F}_{p}: H^{m}(F):=H^{m}\left(G_{F}, \mathbb{F}_{p}\right)$.

The Milnor $K$-groups $K_{m} F$ attached to $F$ are defined as $K_{0} F=\mathbb{Z}$, $K_{1} F=F^{\times}$(the multiplicative group of $F$ ), and for $m>1$, as

$K_{m} F:=\left(F^{\times}\right)^{\otimes m} /\left\langle a_{1} \otimes \cdots \otimes a_{m}: \exists 1 \leq i<j \leq m\right.$ so that $\left.a_{i}+a_{j}=1\right\rangle$.

Here $F^{\times}$is viewed as an abelian group and the tensor product is over 
$\mathbb{Z}$. In fact, we obtain a graded ring,

$$
K_{*}(F)=\bigoplus_{m=0}^{\infty} K_{m}(F),
$$

where the product is induced by tensor products. We define the reduced Milnor $K$-groups as $k_{m} F:=K_{m} F /\langle p\rangle$, and they too form a graded ring which is also a graded vector space over $\mathbb{F}_{p}$. An element of $k_{m} F$ which is represented by $f_{1} \otimes \cdots \otimes f_{m}$ is written in the form $\left\{f_{1}, \ldots, f_{m}\right\}$. For basic properties of Milnor $K$-theory, we refer the reader to $[\mathbf{1 5}, \mathbf{2 9}, \mathbf{4 0}, 49]$.

Now, since $\xi_{p} \in F$, we have $H^{1}(F) \simeq F^{\times} / F^{\times p}$, and so it is obvious that $H^{1}(F)$ and $k_{1} F$ are isomorphic. The norm residue isomorphism says that this is true of higher reduced $K$-groups and cohomology as well, and that this isomorphism respects the underlying ring structures.

The following theorem, proved by Rost and Voevodsky (see [52]), is a substantial advance in Galois cohomology which builds on previous work of Arason, Bass, Bloch, Elman, Jacob, Kato, Lam, Merkurjev, Milnor, Suslin, Tate and others. See also [41] for a very nice survey concerning this theorem in the case $p=2$.

Theorem (Norm residue isomorphism). The rings $k_{*} F$ and $H^{*}(F)$ are isomorphic via the map $h: k_{*} F \rightarrow H^{*}(F)$ defined by $h\left(\left\{f_{1}, \ldots, f_{m}\right\}\right)=$ $\left(f_{1}\right) \cup \cdots \cup\left(f_{m}\right)$.

Although the following proposition can be proved relatively simply, it is the first step in the long journey of proving the norm residue isomorphism. Milnor [29, pages 339-340] stated that this result originated with Bass and Tate.

Proposition 2.1 (Bass-Tate lemma). The map $h: k_{*} F \rightarrow H^{*}(F)$ is well defined.

For this, we need to ensure that the defining relation on the level of $K$-theory maps to a coboundary in cohomology. In other words, we need to show that, if $a \in F^{\times} \backslash\{1\}$ is given, then the element $(a) \cup(1-a)$ is trivial in cohomology. Because the standard proof of 
this result is rather short and interesting, we shall include it here. (See [15, subsection 4.6], [49, Lemma 8.1].)

Proof. Because $\left(b^{p}\right) \cup\left(1-b^{p}\right)=0$ for all $b \in F^{\times}$such that $1 \neq b^{p}$, we shall assume that $a \notin F^{\times p}$. Since we assume $\xi_{p} \in F$, we have the factorization

$$
1-a=\prod_{i=0}^{p-1}\left(1-\xi_{p}^{i} \sqrt[p]{a}\right)
$$

in the field $F(\sqrt[p]{a})$. This factorization, however, is equivalent to taking the norm $N_{F(\sqrt[p]{a}) / F}(1-\sqrt[p]{a})$, and so we have

$$
(a) \cup(1-a)=(a) \cup\left(N_{F(\sqrt[p]{a}) / F}(1-\sqrt[p]{a})\right) .
$$

Now the projection formula (see [39, Proposition 1.5.3 (iv)]) tells us that, when $K / F$ is a Galois extension with $f \in F^{\times}$and $k \in K^{\times}$, then the element $(f) \cup\left(N_{K / F}(k)\right) \in H^{2}(F)$ is equal to $\operatorname{cor}_{K / F}((f) \cup(k))$, where here $\operatorname{cor}_{K / F}: H^{2}(K) \rightarrow H^{2}(F)$ is the corestriction map and the element $(f) \cup(k) \in H^{2}(K)$. Applied to our previous equation, the projection formula gives us

$$
\begin{aligned}
(a) \cup(1-a) & =(a) \cup\left(N_{F(\sqrt[p]{a}) / F}((1-\sqrt[p]{a}))\right) \\
& =\operatorname{cor}_{F(\sqrt[p]{a}) / F}((a) \cup(1-\sqrt[p]{a})) \\
& =\operatorname{cor}_{F(\sqrt[p]{a}) / F}(p(\sqrt[p]{a}) \cup(1-\sqrt[p]{a})) \\
& =\operatorname{cor}_{F(\sqrt[p]{a}) / F}(0)=0 .
\end{aligned}
$$

This proof is certainly elegant, although the critical use of the projection formula prevents us from seeing the result in an "elementary way" (i.e., one which exhibits a given cochain as a coboundary). In the case $p=2$, Pfister [40] gave just such an elementary proof of the Bass-Tate lemma. Our goal in this first section is to prove the vanishing of $(a) \cup(1-a)$ in two different ways: first, by exploiting the known connection between the vanishing of $(x) \cup(y)$ and a particular Galois embedding problem concerning $H_{p^{3}}$; and then, by giving an "elementary" proof for $p>2$ akin to Pfister's proof for $p=2$. In fact, in both proofs, certain Galois extensions with Galois group $H_{p^{3}}$ play a key role. If we consider $p=2$ instead, then the Galois extensions to be considered have Galois group either $\mathbb{Z} / 4$ or the dihedral group of order 8 . 


\subsection{A Proof of Bass-Tate via Galois embedding problems.}

The goal of this subsection is to give an alternate proof of the vanishing of $(a) \cup(1-a)$. We shall also assume that $p>2$, as the case $p=2$ is similar and no new insight is obtained when considering this case. For the rest of this section we will assume that $a$ and $1-a$ are independent in $F^{\times} / F^{\times p}$. This is a reasonable assumption as, otherwise, $(1-a) \cup$ (a) vanishes because the cup product is an anticommutative bilinear map $H^{1}\left(G_{F}, \mathbb{F}_{p}\right) \times H^{1}\left(G_{F}, \mathbb{F}_{p}\right) \rightarrow H^{2}\left(G_{F}, F_{p}\right)$ (see [39, Chapter 1, Section 4]). It is possible to shorten our exposition below using [21, subsection 6.6] or alternatively [28, Theorem 3.1] and [42, Chapter 14, Section 4, Exercise 2 (c)]; however, we feel that it is instructive to present a full detailed exposition.

We recall (see [24, page 58]) that, for $x, y \in F^{\times}$, the vanishing of $(x) \cup(y)$ is equivalent to the solvability of the Galois embedding problem:

$$
1 \longrightarrow \mathbb{F}_{p} \longrightarrow H_{p^{3}} \longrightarrow \mathbb{Z} / p \times \mathbb{Z} / p \longrightarrow 1
$$

over $F(\sqrt[p]{x}, \sqrt[p]{y})$. Hence, we will prove that $(a) \cup(1-a)=0$ by finding an explicit $H_{p^{3}}$-extension of $F$ which contains $L:=F(\sqrt[p]{a}, \sqrt[p]{1-a})$ as a quotient. We have already said that the criterion for solving this embedding problem over the field $F(\sqrt[p]{a}, \sqrt[p]{1-a})$ is that

$$
1-a \in N_{F(\sqrt[p]{a}) / F}\left(F(\sqrt[p]{a})^{\times}\right),
$$

and we have already observed this condition in our first proof of BassTate. In a sense, then, we are done. It will be profitable for us to carry the explanation out a bit more completely, however, as this will help us find an explicit representation of $(a) \cup(1-a)$ as a coboundary, and because it is intimately connected to the results we present in subsequent sections.

First, we establish some notation. We know that, for $L=$ $F(\sqrt[p]{a}, \sqrt[p]{1-a})$, we have $\operatorname{Gal}(L / F) \simeq \mathbb{Z} / p \times \mathbb{Z} / p$; we will write $\sigma$ and $\tau$ for generators of this group which are dual to $a$ and $1-a$, respectively (e.g., $\sigma(\sqrt[p]{a})=\xi_{p} \sqrt[p]{a}$, yet $\sigma$ acts trivially on $\left.\sqrt[p]{1-a}\right)$. Now consider $\alpha=1-\sqrt[p]{a} \in F(\sqrt[p]{a})$. We have already seen that

$$
1-a=\prod_{i=0}^{p-1}\left(1-\xi_{p}^{i} \sqrt[p]{a}\right)=N_{F(\sqrt[p]{a}) / F}(\alpha) .
$$


We define $\beta=(\sigma-1)^{p-2}(\alpha)$ and claim that the desired $H_{p^{3}}$-extension of $L / F$ is $L(\sqrt[p]{\beta}) / F$.

First, observe that the $F$-conjugates of $\sqrt[p]{\beta}$ are the $p$ th roots of $\beta$ under the action of $\sigma$ and $\tau$. Since $\beta \in F(\sqrt[p]{a})$, we have $\tau(\beta)=$ $\beta$. On the other hand, the action of $\sigma$ on $\beta$ is nontrivial; because $1+\sigma+\cdots+\sigma^{p-1} \equiv(\sigma-1)^{p-1} \bmod p$, we have

$$
\begin{aligned}
\sigma(\beta) & =\sigma\left((\sigma-1)^{p-2} \alpha\right)=\beta(\sigma-1)^{p-1} \alpha \\
& \equiv \beta N_{F(\sqrt[p]{a}) / F}(\alpha)=\beta(1-a) \bmod F(\sqrt[p]{a})^{\times p} .
\end{aligned}
$$

In either case, though, the $p$ th roots of $\tau(\beta)$ and $\sigma(\beta)$ are contained in $L(\sqrt[p]{\beta})$, and hence $L(\sqrt[p]{\beta})$ is Galois.

To prove

$$
\operatorname{Gal}(L(\sqrt[p]{\beta}) / F) \simeq H_{p^{3}}
$$

begin by noting that this extension is degree $p^{3}$. Hence, all we must do is show that this group is noncommutative and that elements have order at most $p$. Let $\widehat{\sigma}$ and $\widehat{\tau}$ be lifts of $\sigma$ and $\tau$ to $\operatorname{Gal}(L(\sqrt[p]{\beta}) / F)$; it will be enough for us to show that $\widehat{\sigma} \widehat{\tau} \neq \widehat{\tau} \widehat{\sigma}$ and that $\widehat{\sigma}^{p}=\widehat{\tau}^{p}=\mathrm{id}$. To arrive at these results we will only need to investigate actions on $\sqrt[p]{\beta}$ since we already know how $\widehat{\tau}$ and $\widehat{\sigma}$ act on $\sqrt[p]{a}$ and $\sqrt[p]{1-a}$.

We have already seen that $\widehat{\tau}(\beta)=\beta$ and $\widehat{\sigma}(\beta)=\beta(1-a) k^{p}$ for some $k \in F(\sqrt[p]{a})$. By extracting $p$ th roots in these equations, we therefore have

$$
\begin{aligned}
& \widehat{\sigma}(\sqrt[p]{\beta})=\xi_{p}^{x} \sqrt[p]{\beta} \sqrt[p]{1-a} k \\
& \widehat{\tau}(\sqrt[p]{\beta})=\xi_{p}^{y} \sqrt[p]{\beta}
\end{aligned}
$$

for some $x, y \in \mathbb{Z}$. With these identities in hand, it is easy to see that $\widehat{\tau}$ and $\widehat{\sigma}$ do not commute:

$$
\xi_{p} \widehat{\sigma} \widehat{\tau}(\sqrt[p]{\beta})=\widehat{\tau} \widehat{\sigma}(\sqrt[p]{\beta})
$$

Furthermore, by iteratively applying the second part of equation (2.1), one recovers $\widehat{\tau}^{p}(\sqrt[p]{\beta})=\sqrt[p]{\beta}$. Hence, $\widehat{\tau}^{p}=\mathrm{id}$. On the other hand,

$$
\begin{aligned}
\frac{\widehat{\sigma}^{p}(\sqrt[p]{\beta})}{\sqrt[p]{\beta}} & =(\widehat{\sigma}-1)\left(1+\widehat{\sigma}+\widehat{\sigma}^{2}+\cdots+\widehat{\sigma}^{p-1}\right)(\sqrt[p]{\beta}) \\
& =(\widehat{\sigma}-1)\left(\xi_{p}^{z} \sqrt[p]{\left(1+\sigma+\sigma^{2}+\cdots+\sigma^{p-1}\right) \beta}\right)
\end{aligned}
$$




$$
\begin{aligned}
& =(\widehat{\sigma}-1)\left(\xi_{p}^{z} \sqrt[p]{N_{F(\sqrt[p]{a}) / F}(\beta)}\right) \\
& =(\widehat{\sigma}-1)\left(\xi_{p}^{z} \sqrt[p]{1}\right)=1 .
\end{aligned}
$$

In this case, the second-to-last equality comes from the fact that $\beta$ is in the image of $\sigma-1$, and the last equality follows because $\xi_{p} \in F$ by assumption.

2.2. Exhibiting $(1-a) \cup(a)$ explicitly as a coboundary. In [40], Pfister gives a very interesting proof of the vanishing of $(a) \cup(1-a)$ in the case when $p=2$. Pfister's proof uses nothing but basic definitions from Galois cohomology; he shows that $(a) \cup(1-a)=0$ by explicitly exhibiting it as a coboundary. His proof, however, is unmotivated. Where does his choice of coboundary come from? Also, one would like to produce small Galois extensions where one can show the definition of $(a) \cup(1-a)$ and show that it vanishes there. In our proof below, we explicitly exhibit $(1-a) \cup(a)$ as a coboundary; there is no significant difference between this and $(a) \cup(1-a)$, considering the symmetry between $a$ and $1-a$ and the anti-commutative property of the cup product (see [39, Proposition 1.4.4]). We believe that the vanishing of $(1-a) \cup(a)$ is in fact a precursor of the $n$-vanishing Massey conjecture for $n \geq 3$. (See $[\mathbf{9}, \mathbf{1 7}, \mathbf{3 6}, \mathbf{3 7}, \mathbf{3 8}]$ for related material.)

In this section, we provide motivation for Pfister's proof, and in the process, we both extend it to the case $p>2$ and show that $(1-a) \cup(a)$ can be defined on a Galois extension of degree $p^{3}$ on which it vanishes. Although thematically similar, there are some small distinctions between the proof when $p>2$ and $p=2$; for this reason, we shall focus on the case $p>2$ and only indicate the necessary changes for $p=2$. So assume now that $p>2$.

Before diving into the details, we give a short road map for our argument. Let $\mathbf{p}: F_{\text {sep }}^{\times} \rightarrow F_{\text {sep }}^{\times}$be the map $\mathbf{p}(f)=f^{p}$; the kernel of this map is $\left\langle\xi_{p}\right\rangle \simeq \mathbb{Z} / p$, and we write $\eta$ for the associated embedding of $\mathbb{Z} / p$ into $F_{\text {sep }}^{\times}$. The short exact sequence:

$$
1 \longrightarrow \mathbb{Z} / p \stackrel{\eta}{\longrightarrow} F_{\text {sep }}^{\times} \stackrel{\text { p }}{\longrightarrow} F_{\text {sep }}^{\times} \longrightarrow 1
$$


induces the exact sequence on cohomology:

$$
\begin{aligned}
& H^{1}\left(G_{F}, F_{\mathrm{sep}}^{\times}\right) \longrightarrow H^{1}\left(G_{F}, F_{\mathrm{sep}}^{\times}\right) \stackrel{\delta}{\longrightarrow} H^{2}\left(G_{F}, \mathbb{Z} / p\right) \\
& \stackrel{\eta_{*}}{\longrightarrow} H^{2}\left(G_{F}, F_{\mathrm{sep}}^{\times}\right) .
\end{aligned}
$$

Although $H^{1}\left(G_{F}, F_{\text {sep }}^{\times}\right)=\{0\}$ by Hilbert 90 , it is convenient to keep this group in the picture since we need concrete 1-cochains of $G_{F}$ in $F_{\text {sep }}^{\times}$. Using the Galois theory of $p^{3}$-extensions, we produce a 1 -cochain $N \in C^{1}\left(G_{F}, F_{\text {sep }}^{\times}\right)$such that $d^{1}(N)=\eta_{*}((1-a) \cup(a))$, and hence, $\eta_{*}((1-a) \cup(a))=0$ in $H^{2}\left(G_{F}, F_{\text {sep }}^{\times}\right)$. Because $\eta_{*}$ is injective, this implies that $(1-a) \cup(a)=(0) \in H^{2}\left(G_{F}, \mathbb{Z} / p\right)$. However, in order to produce a specific 2 -boundary for the cochain representing $(1-a) \cup(a)$, we chase sequence (2.2) and modify $N$ by another 1-cochain $M$ so that $d^{1}(M)$ is trivial but $N / M$ takes values in $\mathbb{Z} / p$.

As in the previous section, assume that $a$ and $1-a$ represent classes

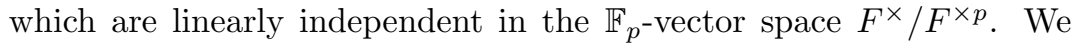
again write $L=F(\sqrt[p]{a}, \sqrt[p]{1-a})$. Thus, $L / F$ is a Galois extension and $\operatorname{Gal}(L / F) \simeq \mathbb{Z} / p \times \mathbb{Z} / p$. We fix a specific primitive $p$ th root of unity $\xi_{p}$. This will give us an isomorphism $\iota:\left\langle\xi_{p}\right\rangle \rightarrow \mathbb{Z} / p$; in this way, we can identify $\left\langle\xi_{p}\right\rangle$ with $\mathbb{Z} / p$ via $\iota$. Following the convention from subsection 2.1, we write $\sigma$ and $\tau$ for the generators of $\operatorname{Gal}(L / F)$ that are dual to $\sqrt[p]{a}$ and $\sqrt[p]{1-a}$, respectively.

Observe that $(1-a) \in H^{1}\left(G_{F}, \mathbb{Z} / p\right)$ is represented by

$$
\gamma \longmapsto \iota(\gamma(\sqrt[p]{1-a}) / \sqrt[p]{1-a})
$$

and, similarly, $(a) \in H^{1}\left(G_{F}, \mathbb{Z} / p\right)$ is represented by

$$
\gamma \longmapsto \iota(\gamma(\sqrt[p]{a}) / \sqrt[p]{a})
$$

Let $\gamma_{1}, \gamma_{2} \in G_{F}$ restrict to $\tau^{i} \sigma^{k}, \tau^{l} \sigma^{j} \in \operatorname{Gal}(L / F)$, respectively; we have (see [39, Proposition 1.4.8]) that $(1-a) \cup(a) \in Z^{2}\left(G_{F}, \mathbb{Z} / p\right)$ is the function

$$
\left(\gamma_{1}, \gamma_{2}\right) \longmapsto \iota\left(\frac{\gamma_{1}(\sqrt[p]{1-a})}{\sqrt[p]{1-a}} \frac{\gamma_{2}(\sqrt[p]{a})}{\sqrt[p]{a}}\right)=i j \bmod p
$$

We now proceed to produce a 1 -cochain $h \in C^{1}\left(G_{F}, \mathbb{Z} / p\right)$ so that $d^{1} h\left(\gamma_{1}, \gamma_{2}\right)=i j \bmod p\left(\right.$ where $d^{1}: C^{1}\left(G_{F}, \mathbb{Z} / p\right) \rightarrow Z^{2}\left(G_{F}, \mathbb{Z} / p\right)$ is 
the usual coboundary map). To begin, set

$$
\alpha=1-\sqrt[p]{a} \in F(\sqrt[p]{a})
$$

and

$$
\beta=\alpha^{p-1} \sigma\left(\alpha^{p-2}\right) \cdots \sigma^{p-2}(\alpha) .
$$

(Note that the element $\beta$ is not identical to the element $\beta$ in subsection 2.1; see also [21, page 162].) Note that, for the element $x=\sqrt[p]{1-a} / \alpha \in L$, we have $\sigma(\beta) / \beta=x^{p}$ and

$$
x \sigma(x) \cdots \sigma^{p-1}(x)=\frac{(\sqrt[p]{1-a})^{p}}{N_{F(\sqrt[p]{a}) / F}(\alpha)}=\frac{1-a}{1-a}=1 .
$$

We also have $\tau(x)=\xi_{p} x$ and $\tau(\beta)=\beta$.

Now let us consider the possible images of $\beta$ under $\operatorname{Gal}(L / F)$. First, one proves by induction that, for any $k \in\{1, \ldots, p-1\}$, we have

$$
\sigma^{k}(\beta)=\left(\sigma^{k-1}(x) \sigma^{k-2}(x) \cdots \sigma(x) x\right)^{p} \beta .
$$

Then, since all elements of $\operatorname{Gal}(L / F)$ can be written in the form $\tau^{i} \sigma^{k}$, and since we already know that $\tau$ acts trivially on $x^{p}$ and $\beta$, this gives all possible images of $\beta$ under $\operatorname{Gal}(L / F)$. In particular, this means that, if $\gamma \in G_{F}$ restricts to $\tau^{i} \sigma^{k}$, then there exists a unique $c \in\{0,1, \ldots, p-1\}$ such that

$$
\gamma(\sqrt[p]{\beta})=\xi_{p}^{c} \sqrt[p]{\beta} \prod_{l=0}^{k-1} \sigma^{l}(x) .
$$

We use this last equation to define two functions from $G_{F}$ to $F_{\text {sep }}^{\times}$:

$$
N(\gamma)=\prod_{l=0}^{k-1} \sigma^{l}(x)
$$

and

$$
M(\gamma)=\frac{\gamma(\sqrt[p]{\beta})}{\sqrt[p]{\beta}}=\xi_{p}^{c} \prod_{l=0}^{k-1} \sigma^{l}(x) .
$$

Note that $M \in Z^{1}\left(G_{F}, F_{\text {sep }}^{\times}\right)$since it is conspicuously in $B^{1}\left(G_{F}, F_{\text {sep }}^{\times}\right)$. Observe that, by using equation (2.3), one can show that the function 
$N \in C^{1}\left(G_{F}, F_{\text {sep }}^{\times}\right)$is well defined. Finally, observe that, since

$$
\beta \equiv(\sigma-1)^{p-2}(\alpha) \in F(\sqrt[p]{a})^{\times} / F(\sqrt[p]{a})^{\times p},
$$

subsection 2.1 tells us that $L(\sqrt[p]{\beta}) / F$ is Galois with $\operatorname{Gal}(L(\sqrt[p]{\beta}) / F)$ $\simeq H_{p^{3}}$. Hence, $M$ and $N$ are defined on the $H_{p^{3}}$-extension $L(\sqrt[p]{\beta}) / F$.

As a consequence, we have a 1-cochain

$$
h=\iota_{*}(N / M) \in C^{1}(\operatorname{Gal}(L(\sqrt[p]{\beta}) / F), \mathbb{Z} / p) .
$$

We show that, as cocycles, we have inf $\left(d^{1} h\right)=(1-a) \cup(a)$. To do this, let us assume that $\gamma_{1}$ and $\gamma_{2}$ are elements of $G_{F}$ with restrictions in $L / F$ of the form $\tau^{i} \sigma^{k}$ and $\tau^{l} \sigma^{j}$. Our goal will be to show inf $\left(d^{1} h\right)\left(\gamma_{1}, \gamma_{2}\right)=$ $i j \bmod p$. Since $M \in B^{1}\left(G_{F}, F_{\text {sep }}\right)$, we know that $d^{1} M$ is trivial. Therefore, we have

$$
\begin{aligned}
\inf \left(d^{1} \frac{N}{M}\right)\left(\gamma_{1}, \gamma_{2}\right) & =\iota\left(\frac{N\left(\gamma_{2}\right)^{\gamma_{1}} N\left(\gamma_{1}\right)}{N\left(\gamma_{1} \gamma_{2}\right)} \frac{M\left(\gamma_{1} \gamma_{2}\right)}{M\left(\gamma_{2}\right)^{\gamma_{1}} M\left(\gamma_{1}\right)}\right) \\
& =\iota\left(\frac{N\left(\gamma_{2}\right)^{\gamma_{1}} N\left(\gamma_{1}\right)}{N\left(\gamma_{1} \gamma_{2}\right)}\right) .
\end{aligned}
$$

By definition, we have

$$
\begin{aligned}
N\left(\gamma_{1}\right) & =\prod_{l=0}^{k-1} \sigma^{l}(x), \\
N\left(\gamma_{2}\right) & =\prod_{l=0}^{j-1} \sigma^{l}(x),
\end{aligned}
$$

and

$$
N\left(\gamma_{1} \gamma_{2}\right)=\prod_{l=0}^{j+k-1} \sigma^{l}(x)
$$

Using the relation $\tau(x)=\xi_{p} x$ and the fact that $\sigma$ and $\tau$ commute in $\operatorname{Gal}(L / F)$, we recover

$$
\frac{N\left(\gamma_{2}\right)^{\gamma_{1}} N\left(\gamma_{1}\right)}{N\left(\gamma_{1} \gamma_{2}\right)}=\left(\prod_{l=0}^{j-1} \sigma^{l}(x)\right)^{\tau^{i} \sigma^{k}}\left(\prod_{l=0}^{k-1} \sigma^{l}(x)\right)\left(\prod_{l=0}^{j+k-1} \sigma^{l}(x)\right)^{-1}
$$




$$
=\left(\prod_{l=0}^{j-1} \sigma^{l}\left(x^{\tau^{i}}\right)\right)^{\sigma^{k}}\left(\prod_{l=0}^{i-1} \sigma^{l}(x)\right)\left(\prod_{l=0}^{j+k-1} \sigma^{l}(x)\right)^{-1}=\xi_{p}^{i j}
$$

This gives the desired result.

The case $p=2$ is nearly identical with the case $p>2$, except that, in this case, one should also consider the cases when $1-a$ and $a$ belong to the same class in $F^{\times} / F^{\times 2}$. Then, $L=F(\sqrt{a}, \sqrt{1-a})=F(\sqrt{a})$ is a quadratic extension and $L(\sqrt{1-\sqrt{a}}) / F$ is cyclic of degree 4 . When $1-a$ and $a$ are independent modulo $F^{\times 2}$, the extension $L(\sqrt{\alpha}) / F$ is a dihedral extension of degree 8.

It is now straightforward to check that the construction above provides a 1-cochain $h \in C^{1}\left(G_{F}, \mathbb{F}_{2}\right)$ such that $d^{1} h=(a) \cup(1-a)$ (with $(a)$ and $(1-a)$ viewed as 1 -cochains and $(a) \cup(1-a)$ viewed as an element of $\left.Z^{2}\left(G_{F}, \mathbb{F}_{2}\right)\right)$. Moreover, in this way we recover the cochain $h$ introduced by Pfister [40, page 275].

3. Recasting the embedding problem. We continue to assume that $p$ is a prime number, but for the rest of the paper we assume that $p>2$. We assume that $F$ is a field and $K / F$ an extension with Galois group isomorphic to $\mathbb{Z} / p^{n}$. We no longer make assumptions on the characteristic of $F$ or on the roots of unity it contains.

Traditionally, theorems concerning the realizability of $H_{p^{3}}$ and $M_{p^{3}}$ as Galois groups are studied from the perspective of embedding problems that arise from group extensions of $\mathbb{Z} / p \times \mathbb{Z} / p$ by $\mathbb{Z} / p$. In the second part of this paper, we revisit the realizability of these groups as Galois groups by studying them instead as a special case of the family of embedding problems:

$$
1 \longrightarrow(\mathbb{Z} / p)^{\oplus \ell} \longrightarrow G \longrightarrow \mathbb{Z} / p^{n} \longrightarrow 1
$$

(Both $H_{p^{3}}$ and $M_{p^{3}}$ occur as short exact sequences for $\ell=2$ and $n=1$.) By studying appropriate $\mathbb{F}_{p}$-vector spaces as modules over Galois groups, we are able to associate the realizations of these groups as Galois groups to the appearance of modules of a certain type within the classical parameterizing spaces of elementary $p$-abelian extensions; by using the recently computed module structures for these spaces, we can revisit the known results concerning these groups from this 
module-theoretic perspective, and furthermore exhibit them as part of a broader phenomena.

We begin by establishing some notation and reminding the reader of some module-theoretic machinery. We write $G_{n}$ for the group $\mathbb{Z} / p^{n}$, and we use $\sigma$ to denote a generator of this group (the particular $n$ corresponding to $\sigma$ will be clear from the context).

$\mathbb{F}_{p}\left[G_{n}\right]$ is a local ring with maximal ideal $\langle\sigma-1\rangle$. We define a homomorphism $\psi: \mathbb{F}_{p}[t] \rightarrow \mathbb{F}_{p}\left[G_{n}\right]$ by $\psi(t)=\sigma-1$. Now $\psi$ is a surjective map, and $t^{p^{n}} \in \operatorname{ker}(\psi)$ because

$$
1=\sigma^{p^{n}}=((\sigma-1)+1)^{p^{n}}=(\sigma-1)^{p^{n}}+1 .
$$

Counting dimensions over $\mathbb{F}_{p}$, we conclude that $\mathbb{F}_{p}[t] /\left\langle t^{p^{n}}\right\rangle \simeq \mathbb{F}_{p}\left[G_{n}\right]$. Hence, we define a "valuation-like" map $v: \mathbb{F}_{p}\left[G_{n}\right] \rightarrow \mathcal{L}_{n}$, where $\mathcal{L}_{n}=\left\{0,1, \ldots, p^{n}-1\right\} \cup\{\infty\}$ is a set endowed with a binary operation * defined by

$$
i * j= \begin{cases}i+j & \text { if } i, j \neq \infty \text { and } i+j \leq p^{n}-1 \\ \infty & \text { if } i=\infty \text { or } j=\infty \text { or } i+j>p^{n}-1\end{cases}
$$

For nonzero $f \in \mathbb{F}_{p}\left[G_{n}\right]$, we will write $v(f)$ for the maximum value $i \in \mathcal{L}_{n} \backslash\{\infty\}$ satisfying $f \in\left\langle(\sigma-1)^{i}\right\rangle$, and we set $v(0)=\infty$. Then we have

$$
v(f g)=v(f) * v(g)
$$

and

$$
v(f+g) \geq \min \{v(f), v(g)\}
$$

(following the usual convention that $\infty>i$ for all $i \in \mathcal{L}_{n} \backslash\{\infty\}$ ). If $M$ is an $\mathbb{F}_{p}\left[G_{n}\right]$-module (written additively) and $\gamma \in M$, then the smallest positive integer $\ell$ such that $(\sigma-1)^{\ell} \gamma=0$ coincides with the $\mathbb{F}_{p}$-dimension of $\langle\gamma\rangle$; we write $\ell(\gamma)$ for this quantity.

If $\gamma_{1}, \gamma_{2} \in M$ and $\mu=\max \left\{\ell\left(\gamma_{1}\right), \ell\left(\gamma_{2}\right)\right\}$ we see that

$$
(\sigma-1)^{\mu}\left(\gamma_{1}+\gamma_{2}\right)=(\sigma-1)^{\mu} \gamma_{1}+(\sigma-1)^{\mu} \gamma_{2}=0
$$

Moreover, if $\ell\left(\gamma_{1}\right)<\ell\left(\gamma_{2}\right)$, then for any $\ell\left(\gamma_{1}\right) \leq v<\ell\left(\gamma_{2}\right)$, we have

$$
(\sigma-1)^{v}\left(\gamma_{1}+\gamma_{2}\right)=(\sigma-1)^{v} \gamma_{1}+(\sigma-1)^{v} \gamma_{2}=(\sigma-1)^{v} \gamma_{2} \neq 0
$$


Hence, we see that

$$
\ell\left(\gamma_{1}+\gamma_{2}\right) \leq \max \left\{\ell\left(\gamma_{1}\right), \ell\left(\gamma_{2}\right)\right\},
$$

with equality if $\ell\left(\gamma_{1}\right) \neq \ell\left(\gamma_{2}\right)$. In what follows, we will refer to this as the ultrametric property.

For $1 \leq \ell \leq p^{n}$, we define the $\mathbb{F}_{p}\left[G_{n}\right]$-module $A_{\ell}$ as $\mathbb{F}_{p}\left[G_{n}\right] /\left\langle(\sigma-1)^{\ell}\right\rangle$; the action of $\sigma$ on $A_{\ell}$ is simply multiplication by $\sigma$. Each of the modules from $\left\{A_{\ell}\right\}_{\ell=1}^{p^{n}}$ is indecomposable, and any indecomposable $\mathbb{F}_{p}\left[G_{n}\right]$-module is isomorphic to some $A_{\ell}$. For any $\mathbb{F}_{p}\left[G_{n}\right]$-module $A$, there is a unique (unordered) collection of positive integers $\left\{\ell_{i}\right\}_{i \in \mathcal{I}}$ (where possibly $\ell_{i}=\ell_{j}$ for $i \neq j$ ) so that $A \simeq \bigoplus_{i \in \mathcal{I}} A_{\ell_{i}}$. (For more details, see [1], [25, subsection 2.3], or [31, subsection 1.1].)

Remark. The $A_{\ell}$ 's are exactly the indecomposable representations of the cyclic group $G_{n}$ over the field $\mathbb{F}_{p}$.

Remark. By a slight abuse of notation, we denote elements of $A_{\ell}$ as elements of $\mathbb{F}_{p}\left[G_{n}\right]$. In these cases, it should be understood that we intend the given element modulo the ideal of $\mathbb{F}_{p}\left[G_{n}\right]$ generated by $(\sigma-1)^{\ell}$.

\subsection{Parameterizing spaces of elementary $p$-abelian exten-} sions. When $K$, and therefore $F$, contains a primitive $p$ th root of unity $\xi_{p}$, Kummer theory tells us that elementary $p$-abelian extensions of $K$ correspond to $\mathbb{F}_{p^{-}}$subspaces of $K^{\times} / K^{\times p}$; these extensions are additionally Galois over $F$ if and only if they are modules over the group $\operatorname{ring} \mathbb{F}_{p}\left[G_{n}\right]$. In this case, we define $J(K)=K^{\times} / K^{\times p}$.

When $K$ has characteristic $p$, Artin-Schreier theory gives a correspondence between elementary $p$-abelian extensions of $K$ and $\mathbb{F}_{p^{-}}$ subspaces of $K / \wp(K)$, where $\wp(K)=\left\{k^{p}-k: k \in K\right\}$. Again, such an extension is Galois over $F$ if and only if the corresponding $\mathbb{F}_{p}$-space is a module over $\mathbb{F}_{p}\left[G_{n}\right]$. In this case, we define $J(K)=K / \wp(K)$.

Finally, if $K$ has characteristic different from $p$ but $\xi_{p} \notin K$, and therefore $\xi_{p} \notin F$, then elementary $p$-abelian extensions of $K$ correspond to $\mathbb{F}_{p}$-subspaces of a particular eigenspace of $K\left(\xi_{p}\right)^{\times} / K\left(\xi_{p}\right)^{\times p}$. Specifically, if $\tau$ is a generator for $\operatorname{Gal}\left(K\left(\xi_{p}\right) / K\right)$ and $\tau\left(\xi_{p}\right)=\xi_{p}^{t}$, then the space parametrizing elementary $p$-abelian extensions of $K$ is the 
subspace on which $\tau$ acts as exponentiation by $t$ (the "t-eigenmodule"). As before, such an extension is Galois over $F$ if and only if the corresponding $\mathbb{F}_{p}$-space is a module over the group ring $\mathbb{F}_{p}\left[G_{n}\right]$ (where here we identify $\operatorname{Gal}(K / F)$ and $\left.\operatorname{Gal}\left(K\left(\xi_{p}\right) / F\left(\xi_{p}\right)\right)\right)$. In this case, we define $J(K)$ as the $t$-eigenmodule of $K\left(\xi_{p}\right)^{\times} / K\left(\xi_{p}\right)^{\times p}$. (These parameterizing spaces are also reviewed in [53].)

It is worth noting that, in this latter case, one can describe a morphism $\mathcal{T}$ which projects subspaces of $K\left(\xi_{p}\right)^{\times} / K\left(\xi_{p}\right)^{\times p}$ to $J(K)$. Let $s=\left[K\left(\xi_{p}\right): K\right]$, and note that $1<s \leq p-1$. Choose $z \in \mathbb{Z}$ so that $z s t^{s-1} \equiv 1 \bmod p$, and, again using the notation $\tau\left(\xi_{p}\right)=\xi_{p}^{t}$, set

$$
\mathcal{T}=z \sum_{i=1}^{s} t^{s-i} \tau^{i-1} \in \mathbb{Z}[\langle\tau\rangle] .
$$

Notice that $(t-\tau) \mathcal{T} \equiv 0 \bmod p$, and so the image of $\mathcal{T}$ is contained in the $t$-eigenspace for $\tau$. Conversely, if an element is in the $t$-eigenspace for $\tau$, then $\mathcal{T}$ acts as the identity. Hence, we have $\mathcal{T}$ projects $\mathbb{F}_{p^{-}}$ subspaces of $K\left(\xi_{p}\right)^{\times} / K\left(\xi_{p}\right)^{\times p}$ onto $J(K)$. (For more details, see [35, Section 4] or [32, Proof of Theorem 2].)

Before moving on, we make a brief comment on notation. Our goal is to prove statements about certain embedding problems within the uniform framework provided by $J(K)$. For this reason, whenever we discuss $J(K)$, we will assume it has an underlying additive structure (and therefore $\operatorname{Gal}(K / F)$ acts multiplicatively).

3.2. Module structures and Galois groups. Because $\operatorname{Gal}(K / F)$ induces an action on $J(K)$, it is natural to consider what this additional structure tells us about elementary $p$-abelian extensions of $K$. The first answer to this question was given by Waterhouse [53], where he considered cyclic submodules of $J(K)$ when char $(K) \neq p$. The question was answered for non-cyclic modules, as well as in the case char $(K)=p$, by $[32,45]$; there the $\mathbb{F}_{p}\left[G_{n}\right]$-module $J(K)$ is exhibited as a parameterizing space for solutions to embedding problems over $K / F$ which arise from extensions of $G_{n}$ by elementary $p$-abelian groups. Since we are focusing on groups related to $H_{p^{3}}$ and $M_{p^{3}}$ in this paper, it will be sufficient for us to focus our attention on cyclic $\mathbb{F}_{p}\left[G_{n}\right]$ submodules. 
Therefore, let $L / K$ be an elementary $p$-abelian extension which corresponds to a cyclic $\mathbb{F}_{p}\left[G_{n}\right]$-submodule $\langle\gamma\rangle \subseteq J(K)$. Then $L / F$ is Galois and $\operatorname{Gal}(L / F)$ is an extension of

$$
\operatorname{Gal}(K / F) \simeq G_{n}
$$

by $\operatorname{Gal}(L / K)$. Using the appropriate parameterizing theory (e.g., Kummer theory if char $(K) \neq p$ and $\left.\xi_{p} \in K\right)$, one can show that there is an equivariant pairing $\operatorname{Gal}(L / K) \times\langle\gamma\rangle \rightarrow \mathbb{F}_{p}$, and hence, $\operatorname{Gal}(L / K)$ is dual to $\langle\gamma\rangle$. One can show that $\mathbb{F}_{p}\left[G_{n}\right]$-modules are self-dual, and so we conclude that $\operatorname{Gal}(L / F)$ is an extension of $\operatorname{Gal}(K / F) \simeq G_{n}$ by $\langle\gamma\rangle$.

With this in mind, we now describe the possible extensions of $G_{n}$ by a cyclic $\mathbb{F}_{p}\left[G_{n}\right]$-module.

Proposition 3.1 ([53, Theorem 2]). There is only one group extension of $G_{n}$ by $A_{p^{n}}$, namely, the semi-direct product $A_{p^{n}} \rtimes G_{n}$. For $1 \leq i<$ $p^{n}$, there are two possible group extensions of $G_{n}$ by $A_{i}$. One of them is the semi-direct product $A_{i} \rtimes G_{n}$, where we have:

$$
\left(f_{1}, \sigma^{j_{1}}\right)\left(f_{2}, \sigma^{j_{2}}\right)=\left(f_{1}+\sigma^{j_{1}} f_{2}, \sigma^{j_{1}+j_{2}}\right) .
$$

The second extension of $G_{n}$ by $A_{i}$ will be written $A_{i} \bullet G_{n}$; the elements of this group again come from $A_{i} \times G_{n}$, but the operation is given by

$$
\left(f_{1}, \sigma^{j_{1}}\right)\left(f_{2}, \sigma^{j_{2}}\right)= \begin{cases}\left(f_{1}+\sigma^{j_{1}} f_{2}, \sigma^{j_{1}+j_{2}}\right) & \text { if } j_{1}+j_{2}<p^{n} \\ \left(f_{1}+\sigma^{j_{1}} f_{2}+(\sigma-1)^{i-1}, \sigma^{j_{1}+j_{2}}\right) & \text { if } j_{1}+j_{2} \geq p^{n}\end{cases}
$$

(Here the numbers $j_{1}$ and $j_{2}$ are taken from $\left\{0, \ldots, p^{n}-1\right\}$. )

Example 3.2. Let $\sigma, \tau \in H_{p^{3}}$ be nontrivial elements which generate $H_{p^{3}}$. Then we have that

$$
[\sigma, \tau]=\sigma \tau \sigma^{-1} \tau^{-1}
$$

is an element of order $p$ which generates $Z\left(H_{p^{3}}\right)$. Now consider the

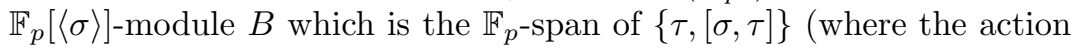
of $\sigma$ is by conjugation). The computation,

$$
(\sigma-1) \cdot \tau=\sigma \tau \sigma^{-1} \tau^{-1}=[\sigma, \tau]
$$


shows that $\langle\tau\rangle=B$ as an $\mathbb{F}_{p}[\langle\sigma\rangle]$-module, and so $B \simeq A_{2}$. Because $\langle\sigma\rangle \cap B=\{1\}$ and $\langle\sigma\rangle \simeq G_{1}$, we conclude that $H_{p^{3}} \simeq A_{2} \rtimes G_{1}$. Phrased slightly differently, this example tells us that, if $N$ is any subgroup of $H_{p^{3}}$ with $|N|=p^{2}$ and $Q:=H_{p^{3}} / N$, then $N \simeq A_{2}$ as an $\mathbb{F}_{p}[Q]$-module and $H_{p^{3}} \simeq N \rtimes Q$.

Now recall that

$$
M_{p^{3}}=\left\langle y, x \mid y^{p^{2}}=x^{p}=1,[x, y]=y^{p}\right\rangle=\langle y\rangle \rtimes\langle x\rangle .
$$

The subgroups $\left\langle y^{k} x\right\rangle$ for $k \in\{1, \ldots, p-1\}$, together with the subgroup $\langle y\rangle$, provide $p$ distinct subgroups isomorphic to $\mathbb{Z} / p^{2}$. On the other hand, we claim that the subgroup $N=\left\langle y^{p}, x\right\rangle$ can be the only subgroup of $M_{p^{3}}$ isomorphic to $\mathbb{Z} / p \times \mathbb{Z} / p$. To see this, note that the $p$ subgroups isomorphic to $\mathbb{Z} / p^{2}$ provide us with $p \cdot \phi\left(p^{2}\right)=p^{3}-p^{2}$ elements of order $p^{2}$, and so the number of elements of order less than $p^{2}$ within $M_{p^{3}}$ is at most $p^{2}$. Since $N$ already contains $p^{2}$ elements of this type, it can be the only subgroup isomorphic to $\mathbb{Z} / p \times \mathbb{Z} / p$. On the other hand, a direct computation shows that $\left[y^{p}, x\right]=1$, so that $N \simeq \mathbb{Z} / p \times \mathbb{Z} / p$.

Now let

$$
Q=M_{p^{3}} / N=\langle y N\rangle \simeq G_{1} .
$$

We have that $N$ is generated by $x$ under the action of $y N$, and hence, $N \simeq A_{2}$ as an $\mathbb{F}_{p}\left[G_{1}\right]$-module. In this case, however, one has $M_{p^{3}} \simeq A_{2} \bullet G_{1}$ (one can verify this directly, or simply note that $A_{2} \rtimes G_{1}$ has no elements of order $p^{2}$ ).

Example 3.3. Let $n \geq 3$, and consider the group $A_{2} \bullet G_{n-2}$. Using the notation from Proposition 3.1, we have

$$
\begin{aligned}
& (0, \sigma)^{p^{n-2}}=(\sigma-1,1) \\
& (0, \sigma)^{p^{n-1}}=\left((0, \sigma)^{p^{n-2}}\right)^{p}=(\sigma-1,1)^{p}=(0,1) .
\end{aligned}
$$

Hence, $A_{2} \bullet G_{n-2}$ is a nonabelian $p$-group with order $p^{n}$ that contains an element of order $p^{n-1}$. Since we have already observed that there is only one such $p$-group, which we previously called $M_{p^{n}}$, we must have $M_{p^{n}} \simeq A_{2} \bullet G_{n-2}$.

If $L / K$ corresponds to $\langle\gamma\rangle$, all that is left to determine is which extension of $G_{n}$ by $\langle\gamma\rangle$ corresponds to $\operatorname{Gal}(L / F)$. To do this, one uses 
the so-called index function. The index is a function

$$
e: J(K) \cap \operatorname{ker}\left((\sigma-1)^{p^{n}-1}\right) \rightarrow \mathbb{F}_{p},
$$

defined by

$$
e(\gamma)= \begin{cases}\sqrt[p]{N_{\widehat{K} / \widehat{F}}(\gamma)} & \text { if } \operatorname{char}(K) \neq p, \\ (\sigma-1) \rho\left(\operatorname{Tr}_{K / F}(\gamma)\right) & \text { if } \operatorname{char}(K)=p .\end{cases}
$$

(In the first case we have identified $\mu_{p}$ with $\mathbb{F}_{p}$ by selecting a particular root of unity $\xi_{p}$ to act as a generator of $\mu_{p}$.) With the index in hand, we obtain the following.

Proposition 3.4 ([45], Theorem 4.4). Suppose $\operatorname{Gal}(K / F) \simeq G_{n}$. Solutions to the embedding problem $A_{p^{n}} \rtimes G_{n} \rightarrow G_{n}$ are in correspondence with submodules $\langle\gamma\rangle \subseteq J(K)$ such that $\ell(\gamma)=p^{n}$. For $i<p^{n}$, solutions to the embedding problem

$$
A_{i} \rtimes G_{n} \rightarrow G_{n}
$$

over $K / F$ are in correspondence with the submodules $\langle\gamma\rangle \subseteq J(K)$ such that $\ell(\gamma)=i$ and $e(\gamma)=0$; solutions to the embedding problem

$$
A_{i} \bullet G_{n} \rightarrow G_{n}
$$

over $K / F$ are in correspondence with the submodules $\langle\gamma\rangle \subseteq J(K)$ such that $\ell(\gamma)=i$ and $e(\gamma) \neq 0$.

Example 3.5. Suppose that $L / F$ has $\operatorname{Gal}(L / F) \simeq H_{p^{3}}$, and let $K / F$ be any $\mathbb{Z} / p$-subextension. Then, $L / K$ is Galois with $\operatorname{Gal}(L / K) \simeq$ $\mathbb{Z} / p \times \mathbb{Z} / p$, and hence, $L$ corresponds to some submodule $M \subseteq J(K)$. Since $\operatorname{Gal}(L / F)$ is nonabelian, it must be the case that $M \nsucceq A_{1} \oplus A_{1}$, and so $M \simeq A_{2}$. If $\langle\gamma\rangle=M$, we must have $e(\gamma)=0$ by Proposition 3.4. Conversely, if $K / F$ is an extension with $\operatorname{Gal}(K / F) \simeq \mathbb{Z} / p$ and $\langle\gamma\rangle \subseteq$ $J(K)$ satisfies $\ell(\gamma)=2$ and $e(\gamma)=0$, then $\langle\gamma\rangle$ corresponds to an extension $L / K$ with $\operatorname{Gal}(L / F) \simeq H_{p^{3}}$.

Suppose now that $L / F$ has $\operatorname{Gal}(L / F) \simeq M_{p^{3}}$. Then there is a unique subextension $K / F$ with $\operatorname{Gal}(K / F) \simeq \mathbb{Z} / p$ and $\operatorname{Gal}(L / K) \simeq \mathbb{Z} / p \times \mathbb{Z} / p$. Within $J(K)$, there exists a submodule $M$ corresponding to $L / K$, and again, it must be the case that $M=\langle\gamma\rangle$ with $\ell(\gamma)=2$ and $e(\gamma) \neq 0$. Conversely, if $K / F$ is an extension with $\operatorname{Gal}(K / F) \simeq \mathbb{Z} / p$ 
and $\langle\gamma\rangle \subseteq J(K)$ satisfies $\ell(\gamma)=2$ and $e(\gamma) \neq 0$, then $\langle\gamma\rangle$ corresponds to an extension $L / F$ containing $K$ with $\operatorname{Gal}(L / F) \simeq M_{p^{3}}$.

The structure of $J(K)$ was computed when $\xi_{p} \in K$ [31, Theorem 2], when $\xi_{p} \notin K$ but char $(K) \neq p[32$, Theorem 2], and when $\operatorname{char}(K)=p$ [45, Proposition 6.2]. (Note that, in the case of $\operatorname{char}(K)=p$ and $\xi_{p} \notin F$, there is a module decomposition for $K^{\times} / K^{\times p}$ provided by [31, Theorem 1]; in this case, however, $J(K) \neq K^{\times} / K^{\times p}$, so this is not the decomposition we provide below.) Although there are some distinctions between the structure of $J(K)$ in these cases, certain qualitative information about these modules is common in all cases. We summarize the important characteristics in the following.

Proposition 3.6. If $\operatorname{Gal}(K / F) \simeq G_{n}$, then $J(K)=\langle\chi\rangle \oplus \bigoplus_{i=0}^{n} Y_{i}$, where:

- $\ell(\chi)=p^{i(K / F)}+1$ for some $i(K / F) \in\{-\infty, 0,1, \cdots, n-1\}$, and $e(\chi) \neq 0$; and

- for each $0 \leq i \leq n$, there exists $\mathfrak{d}_{i}$ so that $Y_{i} \simeq \bigoplus_{\mathfrak{d}_{i}} \mathbb{F}_{p}\left[G_{i}\right]$, and if $i<n$, then $Y_{i} \subseteq \operatorname{ker} e$.

The invariant $i(K / F)$ from this theorem has a variety of interpretations, although there are two that are important for our purposes. The first has an embedding problem flavor. If $K / F$ embeds in a cyclic extension of degree $p^{n+1}$, then $i(K / F)=-\infty$. Otherwise, write $K_{i}$ for the subextension of degree $p^{i}$ over $F$, and choose $s$ minimally so that $K / K_{s}$ embeds in a cyclic extension of degree $p^{n-s+1}$; then $i(K / F)=s-1$. Note, in particular, that $i(K / F)=-\infty$ whenever char $(K)=p$, since Witt's [55] theorem on embedding problems in characteristic $p$ tells us that any $\mathbb{Z} / p^{n}$-extension embeds in a $\mathbb{Z} / p^{n+1}$-extension in this setting. The second interpretation of $i(K / F)$ concerns the dimensions of modules generated by elements with nontrivial index: if $e(\gamma) \neq 0$, then $\ell(\gamma) \geq \ell(\chi)=p^{i(K / F)}+1$.

In light of this correspondence, one of the immediate observations to make from Proposition 3.6 is the following.

Corollary 3.7 (cf. [11, Proposition 10.2]). If $K / F$ is a $\mathbb{Z} / p$-extension, then either $\mathbb{Z} / p^{2} \rightarrow \mathbb{Z} / p$ or $M_{p^{3}} \rightarrow \mathbb{Z} / p$ is solvable over $K / F$. 
More generally, if $K / F$ is a $\mathbb{Z} / p^{n}$-extension, then, for some $i \in$ $\{-\infty, 0,1, \ldots, n-1\}$, the embedding problem $A_{p^{i}+1} \bullet \mathbb{Z} / p^{n} \rightarrow \mathbb{Z} / p^{n}$ is solvable over $K / F$.

Proof. By Proposition 3.6, there exists an element $\chi \in J(K)$ so that $e(\chi) \neq 0$ and $\ell(\chi)=p^{i(K / F)}+1$ for some $i(K / F) \in\{-\infty, 0,1, \ldots$, $n-1\}$. By Proposition 3.4, this module corresponds to a solution to the embedding problem $A_{p^{i(K / F)+1}} \bullet G_{n} \rightarrow G_{n}$.

Corollary 3.8. If $K / F$ is a $\mathbb{Z} / p^{n}$-extension so that, for some $j>i$, both $A_{i} \bullet \mathbb{Z} / p^{n} \rightarrow \mathbb{Z} / p^{n}$ and $A_{j} \bullet \mathbb{Z} / p^{n} \rightarrow \mathbb{Z} / p^{n}$ are solvable over $K / F$, then $A_{j} \rtimes \mathbb{Z} / p^{n} \rightarrow \mathbb{Z} / p^{n}$ is also solvable over $K / F$.

Proof. Solutions to the embedding problems $A_{i} \bullet G_{n} \rightarrow G_{n}$ and $A_{j} \bullet$ $G_{n} \rightarrow G_{n}$ correspond to elements $\gamma_{i}, \gamma_{j} \in J(K)$ with nontrivial index and satisfying $\ell\left(\gamma_{i}\right)=i$ and $\ell\left(\gamma_{j}\right)=j$. By choosing an appropriate $c \in \mathbb{Z} \backslash p \mathbb{Z}$, one has $e\left(c \gamma_{i}+\gamma_{j}\right)=0$; furthermore, $\ell\left(c \gamma_{i}+\gamma_{j}\right)=j$ by the ultrametric property. Hence, $\left\langle c \gamma_{i}+\gamma_{j}\right\rangle$ corresponds to a solution to $A_{j} \rtimes G_{n} \rightarrow G_{n}$.

Remark. One cannot make this statement stronger by saying that the appearance of $A_{i} \bullet G_{n}$ and $A_{j} \bullet G_{n}$ over a field $F$ forces the appearance of $A_{j} \rtimes G_{n}$ over $F$ since there are fields $F$ which admit both $\mathbb{Z} / p^{2} \simeq A_{1} \bullet G_{1^{-}}$and $M_{p^{3}} \simeq A_{2} \bullet G_{1}$-extensions, but which do not admit an $H_{p^{3}} \simeq A_{2} \rtimes G_{1}$-extension. See, for example, [6, page 167].

4. Automatic realizations related to $H_{p^{3}} \Rightarrow M_{p^{3}}$. The last two results show that the appearance of certain groups as Galois groups over a field $F$ can force the appearance of other groups as Galois groups over $F$ as well in a non-trivial way. In this section, we will consider other results in this vein. For a group $G$ and a field $F$, we write $\nu(G, F)$ for the number of extensions $L / F$ with $\operatorname{Gal}(L / F) \simeq G$ in a fixed algebraic closure of $F$. In the same way, $\nu(G \rightarrow Q, K / F)$ counts the number of solutions $L / F$ to a given embedding problem $G \rightarrow Q$ over $K / F$.

A group $G$ is said to automatically realize a group $H$ if $\nu(G, F)>1$ implies $\nu(H, F)>1$ for any field $F$. The classic automatic realization theorem is Whaples's result [54] that, if $p$ is an odd prime number, then $\mathbb{Z} / p^{i}$ automatically realizes $\mathbb{Z} / p^{j}$ for all $i<j$. One can also 
consider automatic realizations for embedding problems: $G \rightarrow Q$ is said to automatically realize $H \rightarrow Q$ if $\nu(G \rightarrow Q, K / F) \geq 1$ implies $\nu(H \rightarrow Q, K / F) \geq 1$.

Brattström was the first to consider automatic realizations between $H_{p^{3}}$ and $M_{p^{3}}$ [6]. She showed that $H_{p^{3}}$ automatically realizes $M_{p^{3}}$ [6, Theorem 2] and that $M_{p^{3}}$ does not automatically realize $H_{p^{3}}$ in general [6, page 167]. (The fact that $H_{p^{3}}$ automatically realizes $M_{p^{3}}$ was also proved in a different way in [11, Corollary 12].) However, she does argue that the solvability of the embedding problem $M_{p^{3}} \rightarrow G_{1}$ over a field $K / F$ will imply the solvability of the embedding problem $H_{p^{3}} \rightarrow G_{1}$ over $K / F$ if either $\operatorname{char}(K)=p$ or $\xi_{p} \in N_{K / F}\left(K^{\times}\right)[\mathbf{6}$, Theorem 2]. (There are some other known automatic realization results associated with $H_{p^{3}}$ and $M_{p^{3}}$. For instance, in [20, Theorem 1.4A], it was observed that, for any finite group $G$, the group $H_{p^{3}} \times G$ automatically realizes $M_{p^{3}} \times G$. In [20, Proposition 1.5], it was also observed that, if

$$
A=\left\langle x, y \mid x^{p^{2}}=y^{p^{2}}=1, x y=y x^{1+p}\right\rangle,
$$

then $M_{p^{3}}$ automatically realizes $A$.)

By interpreting $H_{p^{3}}$ as $A_{2} \rtimes G_{1}$ and $M_{p^{3}}$ as $A_{2} \bullet G_{1}$, we now show Brattström's results can be viewed from the perspectives of Propositions 3.4 and 3.6. Indeed, if the embedding problem $A_{2} \rtimes G_{1} \rightarrow$ $G_{1}$ is solvable, then there exists $\langle\gamma\rangle \subseteq J(K)$ with $\ell(\gamma)=2$ and $e(\gamma)=0$. Using the notation of Proposition 3.6, if $\ell(\chi)=2$, then $\langle\chi\rangle$ corresponds to a solution to $A_{2} \bullet G_{1} \rightarrow G_{1}$, and we are done. Otherwise, $\ell(\chi)=1$, and so $\ell(\gamma+\chi)=2$ by the ultrametric property. Since $e(\gamma+\chi)=e(\chi) \neq 0$, it therefore follows that $\langle\gamma+\chi\rangle$ corresponds to a solution to $A_{2} \bullet G_{1} \rightarrow G_{1}$ over $K / F$.

On the other hand, suppose that $L / F$ is an $M_{p^{3} \text {-extension, and let }}$ $K / F$ be the unique $\mathbb{Z} / p$-subextension. If we assume $\xi_{p} \in N_{K / F}\left(K^{\times}\right)$, then by Albert's famous result, we know that $K / F$ embeds in a $\mathbb{Z} / p^{2}$ extension, whereas if $\operatorname{char}(F)=p$, then it is Witt's theorem which tells us that $K / F$ embeds in a $\mathbb{Z} / p^{2}$-extension. In either case, we conclude that $\chi$ from Proposition 3.6 must satisfy $\ell(\chi)=1$. Now, let $\langle\gamma\rangle$ correspond to the given $M_{p^{3}}$ extension. Then $\ell(\gamma)=2$ and $e(\gamma) \neq 0$. By choosing an appropriate $c \in \mathbb{Z} \backslash p \mathbb{Z}$, one has $e(\gamma+c \chi)=0$ 
and $\ell(\gamma+c \chi)=2$. Hence, this element corresponds to a solution to $A_{2} \rtimes G_{1} \rightarrow G_{1}$.

Using this same line of reasoning we fit this result into a family of similar results which we phrase in the slightly stronger language of automatic realizations of embedding problems.

Proposition 4.1. We have the following automatic realization results:

(1) $A_{\ell} \rtimes G_{n} \rightarrow G_{n}$ automatically realizes $A_{\ell+1} \rtimes G_{n} \rightarrow G_{n}$ for $\ell \neq p^{k}$ with $k \in\{0,1, \ldots, n-1\}$;

(2) $A_{\ell} \bullet G_{n} \rightarrow G_{n}$ automatically realizes $A_{\ell} \rtimes G_{n} \rightarrow G_{n}$ for $\ell \neq p^{k}+1$ with $k \in\{0,1, \ldots, n-1\}$;

(3) $A_{\ell} \bullet G_{n} \rightarrow G_{n}$ automatically realizes $A_{\ell-1} \bullet G_{n} \rightarrow G_{n}$ for $\ell \neq p^{k}+1$ with $k \in\{0,1, \ldots, n-1\}$; and

(4) $A_{p^{n-1}+1} \rtimes G_{n} \rightarrow G_{n}$ automatically realizes $A_{p^{n-1}+k} \bullet G_{n} \rightarrow G_{n}$ for $1 \leq k<p^{n}-p^{n-1}$.

Proof. Proposition 4.1 (1) was essentially the subject of [32], but we again prove the result here. A solution to the embedding problem $A_{\ell} \rtimes G_{n} \rightarrow G_{n}$ corresponds to a submodule $\langle\gamma\rangle \subseteq J(K)$ with $\ell(\gamma)=\ell$ and $e(\gamma)=0$. By Proposition 3.6, we can find an $\mathbb{F}_{p}\left[G_{n}\right]$-basis $\{\chi\} \cup\left\{\alpha_{i}\right\}_{i \in \mathcal{I}}$ for $J(K)$ so that $e(\chi) \neq 0$ and $\ell(\chi)=p^{i}(K / F)+1$, and so that, for all $i \in \mathcal{I}$, we have $\ell\left(\alpha_{i}\right)=p^{\ell_{i}}$ for $\ell_{i} \in\{0, \ldots, n\}$ and $e\left(\alpha_{i}\right)=0$ when $\ell\left(\alpha_{i}\right)<p^{n}$. Express

$$
\gamma=f \chi+\sum_{i \in \mathcal{I}} f_{i} \alpha_{i}
$$

with $f, f_{i} \in \mathbb{F}_{p}\left[G_{n}\right]$; since $e(\gamma)=0$, it must be the case that $f \in\langle\sigma-1\rangle$. Likewise, since $\ell(\gamma)=\ell<p^{n}$, we must have $f_{i} \in\langle\sigma-1\rangle$ for all $i \in \mathcal{I}$ such that $\ell\left(\alpha_{i}\right)=p^{n}$. Now we have $\ell(\gamma)=\max \left\{\ell(f \chi),\left\{\ell\left(f_{i} \alpha_{i}\right)\right.\right.$ : $i \in \mathcal{I}\}\}$ using the ultrametric property together with the $\mathbb{F}_{p}\left[G_{n}\right]$ independence of the set $\{\chi\} \cup\left\{\alpha_{i}\right\}_{i \in \mathcal{I}}$.

We consider two cases. If $\ell(\gamma)=\ell(f \chi)$, then since $\ell \neq p^{k}$, for any $k \in\{0,1, \ldots, n-1\}$ and $\ell(f \chi)=p^{i(K / F)}+1-v(f)$, we must have $v(f) \geq 2$. Hence, $\ell\left((\sigma-1)^{v(f)-1} \chi\right)=\ell+1$ and $e\left((\sigma-1)^{v(f)-1} \chi\right)=0$. Therefore, $A_{\ell+1} \rtimes G_{n} \rightarrow G_{n}$ has a solution.

On the other hand, if $\ell(\gamma)=\ell\left(f_{i} \alpha_{i}\right)$ for some $i \in \mathcal{I}$, then since $\ell\left(\alpha_{i}\right)=p^{\ell_{i}}$, it must be the case that $v\left(f_{i}\right) \geq 1$. But then $\ell((\sigma$ 
$\left.-1)^{v\left(f_{i}\right)-1} \alpha_{i}\right)=\ell+1$ and $e\left((\sigma-1)^{v\left(f_{i}\right)-1} \alpha_{i}\right)=0$. Again, we have a solution to $A_{\ell+1} \rtimes G_{n} \rightarrow G_{n}$.

Proposition 4.1 (2) has two potential proofs. From the grouptheoretic perspective, $A_{\ell-1} \rtimes G_{n}$ is a quotient of $A_{\ell} \bullet G_{n}$, and hence $A_{\ell} \bullet G_{n} \rightarrow G_{n}$ trivially automatically realizes $A_{\ell-1} \rtimes G_{n} \rightarrow G_{n}$. Then item (1) tells us that $A_{\ell-1} \rtimes G_{n} \rightarrow G_{n}$ automatically realizes $A_{\ell} \rtimes G_{n} \rightarrow G_{n}$.

Alternatively, one could prove this result module-theoretically. In this case, a solution to $A_{\ell} \bullet G_{n} \rightarrow G_{n}$ implies the existence of a submodule $\langle\gamma\rangle \subseteq J(K)$ with $\ell(\gamma)=\ell$ and $e(\gamma) \neq 0$. Choose an appropriate value $c \in \mathbb{Z} \backslash p \mathbb{Z}$ so that $e(c \chi+\gamma)=0$, and note that $\ell(c \chi+\gamma)=\ell(\gamma)$ by the ultrametric property (recall that, if $e(\gamma) \neq 0$, then $\ell(\gamma) \geq \ell(\chi)$, and our hypothesis forces this inequality to be strict). Hence, $\langle c \chi+\gamma\rangle$ corresponds to a solution to $A_{\ell} \rtimes G_{n} \rightarrow G_{n}$.

For the proof of Proposition 4.1 (3), suppose that $\langle\gamma\rangle \subseteq J(K)$ corresponds to a solution to the embedding problem $A_{\ell} \bullet G_{n} \rightarrow G_{n}$. The module-theoretic proof of item (2) gives us a module $\langle c \chi+\gamma\rangle$ with $e(c \chi+\gamma)=0$ and $\ell=\ell(c \chi+\gamma)>\ell(\chi)$. If $\ell(\chi)=\ell-1$, then $\langle\chi\rangle$ corresponds to a solution to the embedding problem $A_{\ell-1} \bullet G_{n} \rightarrow G_{n}$. Otherwise, $\ell(\chi+(\sigma-1)(c \chi+\gamma))=\ell-1$, and of course, this module is generated by an element of nontrivial index. Hence, it corresponds to a solution to the embedding problem $A_{\ell-1} \bullet G_{n} \rightarrow G_{n}$.

Finally, to prove Proposition 4.1 (4), we use item (1) to conclude that there is a solution to $A_{p^{n-1}+k} \rtimes G_{n} \rightarrow G_{n}$ over $K / F$. Write $\langle\gamma\rangle \subseteq J(K)$ for the module that corresponds to a solution to the embedding problem $A_{p^{n-1}+k} \rtimes G_{n} \rightarrow G_{n}$. Obviously, $e(\chi+\gamma) \neq 0$, and, if we can show $\ell(\chi+\gamma)=p^{n-1}+k$, then this module will correspond to a solution to the embedding problem $A_{p^{n-1}+k} \bullet G_{n} \rightarrow G_{n}$. We know that $\ell(\chi+\gamma) \leq \max \left\{p^{n-1}+k, p^{i(K / F)}+1\right\}=p^{n-1}+k$ with equality if either $k>1$ or $i(K / F) \neq n-1$. Hence, $\ell(\chi+\gamma)<p^{n-1}+k$ implies both $k=1$ and $i(K / F)=n-1$. But, since $e(\chi+\gamma) \neq 0$ and $\chi$ is an element of minimal length amongst elements of non-trivial index, we must also have

$$
p^{n-1}+1>\ell(\chi+\gamma) \geq \ell(\chi)=p^{n-1}+1,
$$

a contradiction. 
Finally, we give a proposition which builds on Proposition 4.1 (4) but doesn't require the underlying $\mathbb{F}_{p}\left[G_{n}\right]$-module to have such a large dimension. Recall that $K_{i}$ denotes the intermediate field in the extension $K / F$ such that $\left[K_{i}: F\right]=p^{i}$.

Proposition 4.2. Let $i \in\{0,1, \ldots, n-1\}$ be given. If the embedding problems $G_{n-i} \rightarrow G_{n-i-1}$ over $K / K_{i+1}$ and $A_{p^{i}+1} \rtimes G_{n} \rightarrow G_{n}$ over $K / F$ are both solvable, then the embedding problem $A_{p^{i}+k} \bullet G_{n} \rightarrow G_{n}$ is also solvable over $K / F$ for $1 \leq k \leq p^{i+1}-p^{i}$.

Proof. The proof of this result is essentially the same as the proof of Proposition 4.1 (4), although our additional hypothesis concerning the solvability of $G_{n-i} \rightarrow G_{n-i-1}$ over $K / K_{i+1}$ tells us that $i(E / F) \leq i$, so that $\ell(\chi) \leq p^{i}+1$. To find a solution to the desired embedding problem, we note that the solvability of $A_{p^{i}+1} \rtimes G_{n} \rightarrow G_{n}$ over $K / F$ implies the solvability of $A_{p^{i}+k} \rtimes G_{n} \rightarrow G_{n}$ over $K / F$. Let $\langle\gamma\rangle$ be a module in $J(K)$ which corresponds to a solution to this embedding problem. Then the module $\langle\gamma+\chi\rangle$ will be a solution to the embedding problem $A_{p^{i}+k} \bullet G_{n} \rightarrow G_{n}$.

5. Enumerating Galois extensions related to $H_{p^{3}}$ and $M_{p^{3}}$. We now shift focus and concentrate on enumeration results related to $H_{p^{3}}$ and $M_{p^{3}}$, particularly within the family of groups $A_{i} \rtimes G_{1}$ and $A_{i} \bullet G_{1}$. One of the results already known in this vein comes from a paper by Brattström ([6, Theorem 5]) where it is shown that

$$
\begin{gathered}
\nu\left(M_{p^{3}}, F\right)=\left(p^{2}-1\right) \nu\left(H_{p^{3}}, F\right) \\
\text { if } \xi_{p^{2}} \in F \text { or } \operatorname{char}(F)=p .
\end{gathered}
$$

Here we present a stronger result that drops the assumption that $\xi_{p^{2}} \in F$ when char $(F) \neq p$ and gives a closed formula for the difference $\nu\left(M_{p^{3}}, F\right)-\left(p^{2}-1\right) \nu\left(H_{p^{3}}, F\right)$.

Before arriving at this result, we will first need to consider the following $\mathbb{F}_{p}$-subspace of $J(F)$ :

$$
\mathfrak{N}= \begin{cases}\frac{N_{F\left(\xi_{p^{2}}\right) / F}\left(F\left(\xi_{p^{2}}\right)^{\times}\right) F^{\times p}}{F^{\times p}} & \text { when } \xi_{p} \in F \\ J(F) & \text { when } \operatorname{char}(F)=p \\ \mathcal{T}\left(\frac{N_{F\left(\xi_{p^{2}}\right) / F\left(\xi_{p}\right)}\left(F\left(\xi_{p^{2}}\right)^{\times}\right) F\left(\xi_{p}\right)^{\times p}}{F\left(\xi_{p}\right)^{\times p}}\right) & \text { when } \operatorname{char}(F) \neq p \text { and } \xi_{p} \notin F .\end{cases}
$$


Recall that, in the latter case, we write $\tau$ for the generator of $\operatorname{Gal}\left(F\left(\xi_{p}\right) / F\right)$, and that $\mathcal{T} \in \mathbb{Z}[\langle\tau\rangle]$. Because $\operatorname{gcd}(|\tau|, p)=1$, we have that

$$
\left(N_{F\left(\xi_{p^{2}}\right) / F\left(\xi_{p}\right)}(\alpha)\right)^{\mathcal{T}}=N_{F\left(\xi_{p^{2}}\right) / F\left(\xi_{p}\right)}\left(\alpha^{\mathcal{T}}\right)
$$

for any $\alpha \in F\left(\xi_{p^{2}}^{\times}\right)$. It follows that $\mathfrak{N}$ is the $t$-eigenspace for $\tau$ within

$$
\frac{N_{F\left(\xi_{p^{2}}\right) / F\left(\xi_{p}\right)}\left(F\left(\xi_{p^{2}}\right)^{\times}\right) F\left(\xi_{p}\right)^{\times p}}{F\left(\xi_{p}\right)^{\times p}} .
$$

The importance of this space is that it parameterizes those elements of $J(F)$ whose corresponding $G_{1}$-extensions admit a solution to the embedding problem $G_{2} \rightarrow G_{1}$.

Proposition 5.1. Suppose that $f \in J(F)$ corresponds to the $\mathbb{Z} / p$ extension $K / F$. Then the embedding problem $G_{2} \rightarrow G_{1}$ has a solution over $K / F$ if and only if $f \in \mathfrak{N}$.

Proof. This result is [2, Theorem 1] if $\xi_{p} \in F$; when $\xi_{p} \notin F$ and $\operatorname{char}(\mathrm{F}) \neq p$, the result follows by descent. If $\operatorname{char}(\mathrm{F})=p$, then the embedding problem $G_{2} \rightarrow G_{1}$ is always solvable.

We are now prepared to give a generalization of Brattström's result connecting $\nu\left(H_{p^{3}}, F\right)$ and $\nu\left(M_{p^{3}}, F\right)$. In the statement of this theorem we use $\left(\begin{array}{c}n \\ m\end{array}\right)_{p}$ for the $p$-binomial coefficient which counts the number of $m$-dimensional subspaces within an ambient $n$-dimensional $\mathbb{F}_{p}$-space. It is a nice exercise in linear algebra to show that

$$
\left(\begin{array}{l}
n \\
m
\end{array}\right)_{p}=\frac{\left(p^{n}-1\right) \cdots\left(p^{n-m+1}-1\right)}{\left(p^{m}-1\right) \cdots(p-1)}
$$

(see, e.g., [22, Chapter 7]). It is also worth remarking that, by changing the prime $p$ to a variable $q$, one gets the quantum binomial coefficient $\left[\begin{array}{l}n \\ m\end{array}\right]_{q}$ introduced by Gauss. Observe also that $\left(\begin{array}{l}n \\ 1\end{array}\right)_{p}=\left(p^{n}-1\right) /(p-1)$.

Theorem 5.2. Let $p$ be an odd prime, and let $\mathfrak{N}$ be the subspace of $J(F)$ defined above. Then 


$$
\begin{aligned}
& \nu\left(M_{p^{3}}, F\right)=\left(p^{2}-1\right) \nu\left(H_{p^{3}}, F\right) \\
&+\left(\left(\begin{array}{c}
\operatorname{dim}_{\mathbb{F}_{p}} J(F) \\
1
\end{array}\right)_{p}-\left(\begin{array}{c}
\operatorname{dim}_{\mathbb{F}_{p}} \mathfrak{N} \\
1
\end{array}\right)_{p}\right) \frac{|J(F)|}{p^{2}} .
\end{aligned}
$$

Before proving this result, we observe that, when $\operatorname{char}(\mathrm{F}) \neq p$, then $|J(F)|<p^{2}$ is only possible when $p=2$ and $n=1$. Since we are focusing on the case $p>2$, the term $|J(F)| / p^{2}$ is therefore an integer in this case. On the other hand, when $\operatorname{char}(\mathrm{F})=p$, then $|J(F)|<p^{2}$ is possible, but in this case, the term,

$$
\left(\begin{array}{c}
\operatorname{dim} J(F) \\
1
\end{array}\right)_{p}-\left(\begin{array}{c}
\operatorname{dim} \mathfrak{N} \\
1
\end{array}\right)_{p}
$$

vanishes.

Proof. Suppose that $K / F$ is a $G_{1}$-extension. From Proposition 3.4, we know that there is a bijection

$$
\left\{\begin{array}{c}
\mathbb{F}_{p}\left[G_{1}\right] \text {-submodules } \\
M \subseteq J(K) \text { with } \\
M \simeq A_{2} \text { and } M \subseteq \operatorname{ker}(e)
\end{array}\right\} \longleftrightarrow\left\{\begin{array}{c}
\text { Solutions to } \\
H_{p^{3}} \rightarrow G_{1} \\
\text { over } K / F
\end{array}\right\} .
$$

Now, since any given module $M \simeq A_{2}$ satisfies

$$
|\{m \in M: \ell(m)=2\}|=p^{2}-p,
$$

and since any element $m \in J(K) \cap \operatorname{ker}(e)$ with $\ell(m)=2$ generates a submodule corresponding to a solution to $H_{p^{3}} \rightarrow G_{1}$, we get

$$
\nu\left(H_{p^{3}} \rightarrow G_{1}, K / F\right)=\frac{1}{p^{2}-p}|\{\gamma \in J(K) \cap \operatorname{ker}(e): \ell(\gamma)=2\}| .
$$

Since any $H_{p^{3}}$ extension has $p+1$ quotients isomorphic to $\mathbb{Z} / p$, we therefore conclude:

$$
\nu\left(H_{p^{3}}, F\right)=\frac{1}{p+1} \sum_{K / F} \frac{1}{p^{2}-p}|\{\gamma \in J(K) \cap \operatorname{ker}(e): \ell(\gamma)=2\}| .
$$

Now, we will consider $M_{p^{3}}$ extensions, so again, let $K / F$ be a given $G_{1}$-extension. We will fix an element $\chi$ as in Proposition 3.6. According 
to Proposition 3.4, we know that there is a bijection

$$
\left\{\begin{array}{c}
\mathbb{F}_{p}\left[G_{1}\right] \text {-submodules } \\
M \subseteq J(K) \text { with } \\
M \simeq A_{2} \text { and } M \nsubseteq \operatorname{ker}(e)
\end{array}\right\} \longleftrightarrow\left\{\begin{array}{c}
\text { Solutions to } \\
M_{p^{3}} \rightarrow G_{1} \\
\text { over } K / F
\end{array}\right\} .
$$

Again, any module $M \simeq A_{2}$ satisfies $|\{m \in M: \ell(m)=2\}|=p^{2}-p$, and, if the module satisfies $M \nsubseteq \operatorname{ker}(e)$, then it must be that any element from $\{m \in M: \ell(m)=2\}$ has $e(m) \neq 0$. (One can see this in several ways, but here is an embedding problem argument: if there were an element with $\ell(m)=2$ and $e(m)=0$, then $M=\langle m\rangle$ would solve $H_{p^{3}} \rightarrow G_{1}$ instead of $M_{p^{3}} \rightarrow G_{1}$.) Hence, we conclude that

$$
\nu\left(M_{p^{3}} \rightarrow G_{1}, K / F\right)=\frac{1}{p^{2}-p}|\{\alpha \in J(K): \alpha \notin \operatorname{ker}(e), \ell(\alpha)=2\}| .
$$

We claim that $\{\alpha \in J(K): \alpha \notin \operatorname{ker}(e), \ell(\alpha)=2\}$ is equal to

$$
\begin{cases}\bigcup_{c=1}^{p-1} c \chi+\{\gamma \in J(K) \cap \operatorname{ker}(e): \ell(\gamma)=2\} & \text { if } \ell(\chi)=1 \\ \bigcup_{c=1}^{p-1} c \chi+\{\gamma \in J(K) \cap \operatorname{ker}(e): \ell(\gamma)=2\} & \\ \cup \bigcup_{c=1}^{p-1} c \chi+\left(J(K)^{G} \cap \operatorname{ker}(e)\right) & \text { if } \ell(\chi)=2 .\end{cases}
$$

It will be convenient to translate these two conditions into equivalent statements. In the language of Proposition 3.6, the condition $\ell(\chi)=$ 1 is equivalent to $i(K / F)=-\infty$, whereas in the language of the embedding problem, this condition says that $G_{2} \rightarrow G_{1}$ is solvable over $K / F$. On the other hand, $\ell(\chi)=2$ translates to $i(K / F)=0$ in the language of Proposition 3.6, or to the embedding problem statement that $G_{2} \rightarrow G_{1}$ does not have a solution over $K / F$.

If we assume the equality of sets from equation (5.1) for the time being, then, since any given $M_{p^{3}}$-extension has a unique $\mathbb{Z} / p$-quotient, it will follow that:

$$
\begin{aligned}
\nu\left(M_{p^{3}}, F\right) & =\sum_{K / F} \nu\left(M_{p^{3}} \rightarrow G_{1}, K / F\right) \\
& =\sum_{K / F} \frac{1}{p^{2}-p}|\{\alpha \in J(K): \alpha \notin \operatorname{ker}(e), \ell(\alpha)=2\}|
\end{aligned}
$$




$$
\begin{aligned}
& =\frac{p-1}{p^{2}-p}\left(\sum_{K / F}|\{\gamma \in J(K) \cap \operatorname{ker}(e): \ell(\gamma)=2\}|\right. \\
& \left.+\sum_{i(K / F)=0}\left|J(K)^{G} \cap \operatorname{ker}(e)\right|\right) \\
& =\left(p^{2}-1\right) \nu\left(H_{p^{3}}, F\right)+\frac{1}{p} \sum_{i(K / F)=0}\left|J(K)^{G} \cap \operatorname{ker}(e)\right| .
\end{aligned}
$$

We connect this expression to the desired formula for $\nu\left(M_{p^{3}}, F\right)$ by considering two cases. First, suppose $\operatorname{char}(\mathrm{F})=p$. In this case, we have $i(K / F)=-\infty$ for any $G_{1}$-extension $K / F$, and hence, the latter sum is empty. But, in this case, note that the second summand from the desired formula for $\nu\left(M_{p^{3}}, F\right)$ also vanishes since $J(F)=\mathfrak{N}$. Hence, we have the desired result in this case. (Note that, when $\xi_{p^{2}} \in F$, we also have $\mathfrak{N}=J(F)$ so that the latter term vanishes; this proves the other case of Brattström's result.)

Now suppose that $\operatorname{char}(\mathrm{F}) \neq p$. If $K / F$ is a $\mathbb{Z} / p$-extension, let $\iota: J(F) \rightarrow J(K)$ be the map induced by the natural inclusion; from [31, Lemma 8], we have that $J(K)^{G} \cap \operatorname{ker}(e)=\iota(J(F))$, and from the Kummer theory, we have that $|\iota(J(F))|=|J(F)| / p$. Hence, we can continue our chain of equalities by writing:

$$
\begin{aligned}
\nu\left(M_{p^{3}}, F\right)= & \left(p^{2}-1\right) \nu\left(H_{p^{3}}, F\right) \\
& +\frac{|J(F)|}{p^{2}} \mid\left\{K / F: G_{2} \rightarrow G_{1} \text { is not solvable }\right\} \mid .
\end{aligned}
$$

The only thing left to argue, then, is that the number of extensions $K / F$ for which $G_{2} \rightarrow G_{1}$ is not solvable is given by

$$
\left(\begin{array}{c}
\operatorname{dim} J(F) \\
1
\end{array}\right)_{p}-\left(\begin{array}{c}
\operatorname{dim} \mathfrak{N} \\
1
\end{array}\right)_{p}
$$

although this is straightforward given Proposition 5.1: $(\underset{1}{\operatorname{dim} J(F)})_{p}$ counts all $\mathbb{Z} / p$-extensions, and $\left(\begin{array}{c}\operatorname{dim} \mathfrak{N} \\ 1\end{array}\right)_{p}$ counts all $\mathbb{Z} / p$-extensions which admit a solution to the embedding problem $G_{2} \rightarrow G_{1}$.

To finish the proof, then, we simply need to show that $\{\alpha \in J(K)$ : $\alpha \notin \operatorname{ker}(e), \ell(\alpha)=2\}$ is equal to the expression from equation (5.1). Suppose first that $\ell(\chi)=1$. Let $\alpha \in J(K)$ be given which satisfies $e(\alpha) \neq 0$ and $\ell(\alpha)=2$. Then, there exists some $c \in \mathbb{F}_{p}^{\times}$so that 
$\alpha-c \chi \in \operatorname{ker}(e)$, and the ultrametric property gives $\ell(\alpha-c \chi)=2$. This gives one containment. For the other, if $\gamma \in J(K) \cap \operatorname{ker}(e)$ and $\ell(\gamma)=2$, then the ultrametric property tells us that, for any $c \in \mathbb{F}_{p}^{\times}$, we have $\ell(c \chi+\gamma)=2$, and of course, $e(c \chi+\gamma) \neq 0$.

Now suppose that $\ell(\chi)=2$. Let $\alpha \in J(K)$ be given which satisfies $e(\alpha) \neq 0$ and $\ell(\alpha)=2$. Then there exists some $c \in \mathbb{F}_{p}^{\times}$so that $\alpha-c \chi \in \operatorname{ker}(e)$, although this time the ultrametric property only gives $\ell(\alpha-c \chi) \leq 2$. Hence, either $\alpha-c \chi$ has length 2 or $\alpha-c \chi \in J(K)^{G}$. This proves one containment. For the other, the ultrametric property makes it clear that, if $\gamma \in J(K)^{G}$ and $e(\gamma)=0$, then for any $c \in \mathbb{F}_{p}^{\times}$ we have that $e(c \chi+\gamma) \neq 0$ and $\ell(c \chi+\gamma)=2$. We claim that, for $\gamma \in \operatorname{ker}(e)$ with $\ell(\gamma)=2$, it is still true that, for all $c \in \mathbb{F}_{p}^{\times}$, we have $e(c \chi+\gamma) \neq 0$ and $\ell(c \chi+\gamma)=2$. The first statement is clear; for the second, note that if $\ell(c \chi+\gamma)<2$, then this implies that $c \chi+\gamma$ is an element of nontrivial index with length 1 , a contradiction to the fact that $\chi$ is an element of nontrivial index with minimal length.

Notice that, in the previous theorem, we had to be careful in using our methodology to enumerate extensions since our modules naturally parameterize solutions to embedding problems over a given $\mathbb{Z} / p$-extension $K / F$. In the case of $H_{p^{3}}$ extensions of $F$, we had to account for the fact that a given $H_{p^{3}}$ extension of $F$ solves embedding problems over $p+1$ distinct $\mathbb{Z} / p$-extensions of $F$. To adapt the methodology of the previous theorem to a broader class of groups, we will be interested in determining when groups of the form $A_{i} \rtimes G_{n}$ or $A_{i} \bullet G_{n}$ have precisely one normal subgroup which is elementary $p$-abelian and whose quotient is isomorphic $\mathbb{Z} / p^{n}$.

Lemma 5.3. Suppose that $\operatorname{Gal}(L / F) \simeq A_{i} \rtimes G_{n}$ for $i \geq p^{n-1}+2$. Then there is a unique intermediate $\mathbb{Z} / p^{n}$-extension $K / F$ so that $L / K$ is elementary p-abelian. Likewise, if $\operatorname{Gal}(L / F) \simeq A_{i} \bullet G_{n}$ for $i \geq p^{n-1}+1$, then there is a unique intermediate $\mathbb{Z} / p^{n}$-extension $K / F$ so that $L / K$ is elementary p-abelian.

Proof. In each case, it is obvious that $T=\left\{(f, 1): f \in A_{i}\right\}$ is a normal subgroup which is elementary $p$-abelian. Equally clear is that the fixed field is a $\mathbb{Z} / p^{n}$-extension. Before proceeding, we observe that 
the collection of elements in $A_{i} \rtimes G_{n}$ with order $p$ are those of the form $\left(f, \sigma^{j}\right)$ where $p^{n-1} \mid j$.

For the sake of contradiction, suppose that $H \neq T$ is an elementary $p$-abelian normal subgroup of $A_{i} \rtimes G_{n}$ with quotient $\mathbb{Z} / p^{n}$; this forces $|H|=p^{i}$ and $H \backslash T \neq \emptyset$. Choose $\left(f, \sigma^{j}\right) \in H \backslash T$ so that $\ell(f)$ is maximal amongst elements within $H \backslash T$. After taking a suitable power of $\left(f, \sigma^{j}\right)$, if necessary, we can assume that our element is $\left(f, \sigma^{p^{n-1}}\right)$. (Note that $\left(f, \sigma^{j}\right)^{t}=\left(\sum_{i=0}^{t-1} \sigma^{i t} f, \sigma^{j t}\right)$, and that $\sum_{k=0}^{t-1} \sigma^{j k}$ is a unit in $\mathbb{F}_{p}[G]$ for $1 \leq t \leq p-1$, so that $\ell(f)=\ell\left(\sum_{k=0}^{t-1} \sigma^{j k} f\right)$.)

Since $H$ is normal, we know that the commutator $\left[(0, \sigma),\left(f, \sigma^{j}\right)\right]=$ $((\sigma-1) f, 1)$ is an element of $H$; repeating this procedure shows $\{(g, 1): \ell(g)<\ell(f)\} \subseteq H$. By the maximality of $\left(f, \sigma^{p^{n-1}}\right)$, there exists no $\left(g, \sigma^{k}\right) \in H$ with $k \neq p^{n}$ and $\ell(g)>\ell(f)$. We argue that there are also no elements $(g, 1) \in H$ with $\ell(g)>\ell(f)$; otherwise,

$$
(h, 1)\left(f, \sigma^{p^{n-1}}\right)=\left(h+f, \sigma^{p^{n-1}}\right) \in H,
$$

and the ultrametric property gives $\ell(h+f)=\ell(h)$, violating the maximality condition defining $f$. Notice also that, since $((\sigma-1) f, 1) \in$ $H$ and $H$ is assumed to be abelian, we must have

$$
\begin{aligned}
\left(f+(\sigma-1) f, \sigma^{p^{n-1}}\right) & =((\sigma-1) f, 1)\left(f, \sigma^{p^{n-1}}\right) \\
& =\left(f, \sigma^{p^{n-1}}\right)((\sigma-1) f, 1) \\
& =\left(f+\sigma^{p^{n-1}}(\sigma-1) f, \sigma^{p^{n-1}}\right) .
\end{aligned}
$$

It therefore follows that $(\sigma-1) f$ is fixed by $\sigma^{p^{n-1}}$, and so $\ell(f) \leq$ $p^{n-1}+1$.

Now suppose that $H$ contains no elements of the form $\left(g, \sigma^{k}\right)$ with $k \neq p^{n}$ and $\ell(g)<\ell(f)$. Then we have

$$
H=\left\langle\left(f, \sigma^{p^{n-1}}\right),((\sigma-1) f, 1),\left((\sigma-1)^{2} f, 1\right), \cdots,\left((\sigma-1)^{\ell(f)-1} f, 1\right)\right\rangle_{\mathbb{F}_{p}},
$$

and hence, $|H|=p^{\ell(f)} \leq p^{p^{n-1}+1}<p^{i}$, a contradiction.

On the other hand, suppose that $H$ does contain an element $\left(g, \sigma^{k}\right)$ with $k \neq p^{n}$ and $\ell(g)<\ell(f)$. One can argue that $\left(g, \sigma^{k}\right)$ can be chosen to take the form $\left(g, \sigma^{p^{n-1}}\right)$ as before, and, since we have already 
established $(g, 1) \in H$, we conclude that $\left(0, \sigma^{p^{n-1}}\right) \in H$. This gives $\left(f, \sigma^{p^{n-1}}\right)\left(0, \sigma^{p^{n-1}}\right)^{-1}=(f, 1) \in H$ as well. The commutativity of $H$ implies

$$
\left(f, \sigma^{p^{n-1}}\right)=(f, 1)\left(0, \sigma^{p^{n-1}}\right)=\left(0, \sigma^{p^{n-1}}\right)(f, 1)=\left(\sigma^{p^{n-1}} f, \sigma^{j}\right) .
$$

We conclude that $f$ is fixed by the action of $\sigma^{p^{n-1}}$, so that, in fact, $\ell(f) \leq p^{n-1}$ in this case. But then

$$
H=\left\langle(f, 1),((\sigma-1) f, 1), \ldots,\left((\sigma-1)^{\ell(f)-1} f, 1\right),\left(0, \sigma^{p^{n-1}}\right)\right\rangle_{\mathbb{F}_{p}},
$$

contradicting the fact that $i \geq p^{n-1}+2$ in this case. This completes the proof for $A_{i} \rtimes G_{n}$.

Now suppose that $H \neq T$ is an elementary $p$-abelian normal subgroup of $A_{i} \bullet G_{n}$ with quotient $\mathbb{Z} / p^{n}$; this forces $|H|=p^{i}$. Again, we will choose $\left(f, \sigma^{j}\right) \in H \backslash T$ so that $\ell(f)$ is maximal amongst elements within $H \backslash T$, and we again observe that we can assume that this element is of the form $\left(f, \sigma^{p^{n-1}}\right)$. We begin our argument in this case by claiming that $\ell(f)>p^{n}-p^{n-1}$ is necessary. To do so, we first establish some notation. For an integer $a$, we write $\bar{a}$ for the least non-negative residue of $a$ modulo $p^{n}$. For $m \in \mathbb{N}$, we then define $c_{j}(m)$ inductively: $c_{j}(1)=0$, and

$$
c_{j}(m+1)= \begin{cases}c_{j}(m) & \text { if } \overline{m j}+j<p^{n} \\ c_{j}(m)+1 & \text { if } \overline{m j}+j \geq p^{n}\end{cases}
$$

Then one can use induction to show that

$$
\left(f, \sigma^{p^{n-1}}\right)^{m}=\left(\sum_{k=0}^{m-1} \sigma^{k p^{n-1}} f+c_{p^{n-1}}(m)(\sigma-1)^{i-1}, \sigma^{m p^{n-1}}\right) .
$$

Take $m=p$, and observe that $1 \leq c_{p^{n-1}}(p)<p$ (the first inequality follows because $p^{n-1} p \geq p^{n}$, and the latter because $c_{p^{n-1}}(1)=0$ and $c_{p^{n-1}}(m+1)-c_{p^{n-1}}(m) \leq 1$ for all $\left.m\right)$. If $\ell(f) \leq p^{n-1}(p-1)$, this means, contrary to the assumption that $H$ is elementary $p$-abelian, that

$$
\begin{aligned}
\left(f, \sigma^{p^{n-1}}\right)^{p} & =\left(\sum_{k=0}^{p-1} \sigma^{k p^{n-1}} f+c_{p^{n-1}}(p)(\sigma-1)^{i-1}, 1\right) \\
& =\left(\left(\sigma^{p^{n-1}}-1\right)^{p-1} f+c_{p^{n-1}}(p)(\sigma-1)^{i-1}, 1\right) \\
& =\left(c_{p^{n-1}}(p)(\sigma-1)^{i-1}, 1\right) \neq(0,1) .
\end{aligned}
$$


Now observe that $\left[(0, \sigma),\left(f, \sigma^{p^{n-1}}\right)\right] \in H$, and hence, should commute with $\left(f, \sigma^{p^{n-1}}\right)$. But a computation reveals

$$
\left[\left(f, \sigma^{p^{n-1}}\right),\left[(0, \sigma),\left(f, \sigma^{p^{n-1}}\right)\right]\right]=\left((\sigma-1)\left(\sigma^{p^{n-1}}-1\right) f, 1\right) .
$$

Provided either $p>3$ or $n=1$, this element is nontrivial because $\ell(f) \geq p^{n}-p^{n-1}>p^{n-1}+1$, and so $H$ is not commutative.

We will handle the remaining cases $p=3$ and $n=1$ directly. We have the group $A_{i} \bullet \mathbb{Z} / 3 \mathbb{Z}$, where $2 \leq i \leq 3$, and we want to show that this group has a unique $\mathbb{Z} / p$-quotient whose corresponding normal subgroup is $\mathbb{Z} / p \times \mathbb{Z} / p$. When $i=2$, then the group is simply $M_{3^{3}}$, and we already know the result for this group. When $i=3$, then $A_{i} \bullet \mathbb{Z} / 3 \simeq A_{i} \rtimes \mathbb{Z} / 3$, and we have already established the desired result from the first part of this theorem.

Theorem 5.4. For an extension $K / F \simeq \mathbb{Z} / p^{n}$ and $p^{n-1}+2 \leq i<p^{n}$,

$$
\nu\left(A_{i} \bullet G_{n} \rightarrow G_{n}, K / F\right)=(p-1) \nu\left(A_{i} \rtimes G_{n} \rightarrow G_{n}, K / F\right) .
$$

Moreover, for a field $F$ and $p^{n-1}+2 \leq i<p^{n}$,

$$
\nu\left(A_{i} \bullet G_{n}, F\right)=(p-1) \nu\left(A_{i} \rtimes G_{n}, F\right) .
$$

Proof. We begin by noting that Lemma 5.3 tells us that the second statement follows from the first, since a given $A_{i} \rtimes G_{n}$ or $A_{i} \bullet G_{n}$ extension of $F$ has a unique $\mathbb{Z} / p^{n}$-subextension $K / F$ such that $L / K$ is elementary $p$-abelian. Hence, such an extension is parameterized uniquely as a module within $J(K)$, and so we have

$$
\nu\left(A_{i} \rtimes G_{n}, F\right)=\sum_{\operatorname{Gal}(K / F) \simeq G_{n}} \nu\left(A_{i} \rtimes G_{n} \rightarrow G_{n}, K / F\right),
$$

and likewise for the non-semidirect product. Hence, we will focus on proving the first result.

To do so, we note that, using Proposition 3.4 and the fact that any cyclic module of dimension $i$ has $p^{i}-p^{i-1}$ many generators, one has:

$$
\begin{aligned}
\nu\left(A_{i} \rtimes G_{n}\right. & \left.\rightarrow G_{n}, K / F\right)=\frac{1}{p^{i}-p^{i-1}}|\{\gamma \in J(K): \gamma \in \operatorname{ker}(e), \ell(\gamma)=i\}| \\
\nu\left(A_{i} \bullet G_{n}\right. & \left.\rightarrow G_{n}, K / F\right)=\frac{1}{p^{i}-p^{i-1}}|\{\alpha \in J(K): \alpha \notin \operatorname{ker}(e), \ell(\alpha)=i\}| .
\end{aligned}
$$


The desired result will follow if we can show that, for the element $\chi \in J(K)$ from Proposition 3.6, one has

$$
\begin{aligned}
& \{\alpha \in J(K): \alpha \notin \operatorname{ker}(e), \ell(\alpha)=i\} \\
& \quad=\bigcup_{c=1}^{p-1} c \chi+\{\gamma \in J(K): \gamma \in \operatorname{ker}(e), \ell(\gamma)=i\}
\end{aligned}
$$

For this, note that $\ell(\chi)=p^{i(E / F)}+1<i$, and so if $\ell(\gamma)=i$, then the ultrametric property gives $\ell(c \chi+\gamma)=i$. Of course, if $\gamma \in \operatorname{ker}(e)$, as well, then $e(c \chi+\gamma) \neq 0$. This gives one containment. For the other, note that, if $\alpha \notin \operatorname{ker}(e)$, then there exists some $c \in \mathbb{F}_{p}^{\times}$so that $\alpha-c \chi \in \operatorname{ker}(e)$; when $\ell(\alpha)=i$, the ultrametric property again gives $\ell(\alpha-c \chi)=i$, and hence, $\alpha=c \chi+\alpha-c \chi \in c \chi+\{\gamma \in J(K): \gamma \in$ $\operatorname{ker}(e), \ell(\gamma)=i\}$

If one is willing to settle for counting only solutions to embedding problems, one can extend these same ideas to express $\nu\left(A_{\ell} \rtimes\right.$ $\left.G_{1} \rightarrow G_{1}, K / F\right)$ in terms of $\nu\left(H_{p^{3}} \rightarrow G_{1}, K / F\right)$ and $\nu(\mathbb{Z} / p \times \mathbb{Z} / p \rightarrow$ $\mathbb{Z} / p, K / F)$.

Theorem 5.5. For $2 \leq \ell \leq p-1$, and $a \mathbb{Z} / p$-extension $K / F$, we have

$$
\begin{aligned}
& \nu\left(A_{\ell} \rtimes \mathbb{Z} / p \rightarrow \mathbb{Z} / p, K / F\right) \\
= & \nu\left(H_{p^{3}} \rightarrow \mathbb{Z} / p, K / F\right)\left(\frac{1}{p}+\frac{(p-1) \nu\left(H_{p^{3}} \rightarrow \mathbb{Z} / p, K / F\right)}{1+(p-1) \nu(\mathbb{Z} / p \times \mathbb{Z} / p \rightarrow \mathbb{Z} / p, K / F)}\right)^{\ell-2} .
\end{aligned}
$$

Proof. We know that

$$
\nu\left(H_{p^{3}} \rightarrow \mathbb{Z} / p, K / F\right)=\frac{1}{p^{2}-p} \mid\{\gamma \in J(K): \ell(\gamma)=2 \text { and } \gamma \in \operatorname{ker}(e)\} \mid
$$

Likewise, we have

$$
\begin{aligned}
\nu\left(A_{\ell} \rtimes \mathbb{Z} / p \rightarrow\right. & \mathbb{Z} / p, K / F) \\
& =\frac{1}{p^{\ell}-p^{\ell-1}} \mid\{\gamma \in J(K): \ell(\gamma)=\ell \text { and } \gamma \in \operatorname{ker}(e)\} \mid .
\end{aligned}
$$


By Proposition 3.6, we know that the $\mathbb{F}_{p}\left[G_{1}\right]$-structure of $\operatorname{ker}(e)$ is

$$
\operatorname{ker}(e) \simeq \bigoplus^{\mathfrak{d}_{0}} \mathbb{F}_{p} \oplus \bigoplus^{\mathfrak{d}_{1}} \mathbb{F}_{p}\left[G_{1}\right] /(\sigma-1)^{p-2}
$$

Hence, it is relatively simple to see that, for $1 \leq \ell \leq p-1$, we have

$$
\mid\{\gamma \in J: \ell(\gamma) \leq \ell \text { and } \gamma \in \operatorname{ker}(e)\} \mid=p^{\mathfrak{d}_{0}+\ell \mathfrak{d}_{1}} .
$$

Hence, for $2 \leq \ell \leq p-1$, we have

$$
\begin{aligned}
\mid\{\gamma \in J: \ell(\gamma)=\ell \text { and } \gamma \in \operatorname{ker}(e)\} \mid & =p^{\mathfrak{d}_{0}+\ell \mathfrak{d}_{1}}-p^{\mathfrak{d}_{0}+(\ell-1) \mathfrak{d}_{1}} \\
& =p^{\mathfrak{d}_{0}+(\ell-1) \mathfrak{d}_{1}}\left(p^{\mathfrak{d}_{1}}-1\right)
\end{aligned}
$$

and, for $\ell=1$, we get

$$
\mid\{\gamma \in J: \ell(\gamma)=1 \quad \text { and } \quad \gamma \in \operatorname{ker}(e)\} \mid=p^{\mathfrak{d}_{0}+\mathfrak{d}_{1}}-1
$$

Hence, for $2 \leq \ell \leq p-1$, one calculates

$$
\begin{aligned}
\nu\left(A_{\ell} \rtimes \mathbb{Z} / p \rightarrow \mathbb{Z} / p, K / F\right) & =\frac{1}{p^{\ell}-p^{\ell-1}}|\{\gamma \in \operatorname{ker}(e): \ell(\gamma)=\ell\}| \\
& =\frac{1}{p^{\ell-1}(p-1)} p^{\mathfrak{d}_{0}+(\ell-1) \mathfrak{d}_{1}}\left(p^{\mathfrak{d}_{1}}-1\right) \\
& =p^{\mathfrak{d}_{0}+\mathfrak{d}_{1}-1} \frac{p^{\mathfrak{d}_{1}}-1}{p-1}\left(p^{\mathfrak{d}_{1}-1}\right)^{\ell-2} .
\end{aligned}
$$

Of course, the case $\ell=1$ follows in a similar way:

$$
\nu(\mathbb{Z} / p \times \mathbb{Z} / p \rightarrow \mathbb{Z} / p, K / F)=\frac{p^{\mathfrak{d}_{0}+\mathfrak{d}_{1}}-1}{p-1} .
$$

Because it will be particularly useful in a moment, let us also note that

$$
\nu\left(H_{p^{3}} \rightarrow \mathbb{Z} / p, K / F\right)=p^{\mathfrak{d}_{0}+\mathfrak{d}_{1}-1} \frac{p^{\mathfrak{d}_{1}}-1}{p-1} .
$$

One can solve for $p^{\mathfrak{d}_{0}+\mathfrak{d}_{1}}$ and $p^{\mathfrak{d}_{1}}$ using equations (5.3) and (5.4), and ultimately recover

$$
\begin{aligned}
& p^{\mathfrak{d}_{0}+\mathfrak{d}_{1}}=1+(p-1) \nu(\mathbb{Z} / p \times \mathbb{Z} / p \rightarrow \mathbb{Z} / p, K / F) \\
& p^{\mathfrak{d}_{1}}=1+\frac{p(p-1)}{p^{\mathfrak{d}_{0}+\mathfrak{d}_{1}}} \nu\left(H_{p^{3}} \rightarrow \mathbb{Z} / p, K / F\right) \\
& =1+\frac{p(p-1) \nu\left(H_{p^{3}} \rightarrow \mathbb{Z} / p, K / F\right)}{1+(p-1) \nu(\mathbb{Z} / p \times \mathbb{Z} / p \rightarrow \mathbb{Z} / p, K / F)} .
\end{aligned}
$$


With these in hand, we can reexpress (5.2) to satisfy the statement of Proposition 3.6:

$$
\begin{aligned}
& \nu\left(A_{\ell} \rtimes \mathbb{Z} / p \rightarrow \mathbb{Z} / p, K / F\right)=p^{\mathfrak{d}_{0}+\mathfrak{d}_{1}-1} \frac{p^{\mathfrak{d}_{1}}-1}{p-1}\left(p^{\mathfrak{d}_{1}-1}\right)^{\ell-2} \\
= & \nu\left(H_{p^{3}} \nrightarrow \mathbb{Z} / p, K / F\right)\left(\frac{1}{p}+\frac{(p-1) \nu\left(H_{p^{3}} \rightarrow \mathbb{Z} / p, K / F\right)}{1+(p-1) \nu(\mathbb{Z} / p \times \mathbb{Z} / p \rightarrow \mathbb{Z} / p, K / F)}\right)^{\ell-2} .
\end{aligned}
$$

We finish with some realization multiplicity results for groups from the family $A_{i} \rtimes G_{1}$ and $A_{i} \bullet G_{1}$. The realization multiplicity of $G$, written $\nu(G)$, is the minimal positive value for $\nu(G, F)$ as $F$ ranges over all fields; likewise, the realization multiplicity for an embedding problem $G \rightarrow Q$, written $\nu(G \rightarrow Q)$, is the minimal positive value for $\nu(G \rightarrow Q, K / F)$ as $K / F$ ranges over all fields with $\operatorname{Gal}(K / F) \simeq Q$. There are very few realization multiplicity results known for nonabelian $p$-groups; the known results come from [20] and are $\nu\left(M_{p^{3}}\right)=p$, $\nu\left(M_{p^{3}} \times \mathbb{Z} / p\right)=p^{2}-1$ and $\nu\left((\mathbb{Z} / p)^{k} \times H_{p^{3}}\right)=1$ for $k \in \mathbb{Z}_{\geq 0}$.

Example 5.6. Consider a field $F$ with char $(F) \neq p$ and $\operatorname{dim}_{\mathbb{F}_{p}}(J(F))$ $=2$ and such that $G_{F}:=\operatorname{Gal}\left(F_{\text {sep }} / F\right)$ is the free pro- $p$ group on two generators. (Such a field exists as seen in [14, Corollary 23.1.2].) Now we claim that $\xi_{p} \in F$, since otherwise, we would have $F\left(\xi_{p}\right) \subseteq F_{\text {sep }}$ and $1<\left[F\left(\xi_{p}\right): F\right] \leq p-1<p$. But this would imply that $G_{F}$ has a quotient whose order is not a power of $p$, a clear contradiction.

Since $G_{F}$ is a free pro- $p$ group we have $H^{2}(F)=0$, and therefore, $\left(f_{1}\right) \cup\left(f_{2}\right)=0$ for each $f_{1}, f_{2} \in J(F)$. Then we conclude that

$$
f_{1} \in N_{F\left(\sqrt[p]{f_{2}}\right) / F}\left(F\left(\sqrt[p]{f_{2}}\right)\right) \text { for all } f_{1}, f_{2} \in F^{\times} .
$$

Suppose then, that $f_{1}, f_{2}$ are generators for $J(F)$. If we let $\alpha \in F\left(\sqrt[p]{f_{2}}\right)$ be given so that

$$
N_{F\left(\sqrt[p]{f_{2}}\right) / F}(\alpha)=f_{1},
$$

and if we write $\sigma$ for the generator of the $G_{1}$-extension $F\left(\sqrt[p]{f_{2}}\right) / F$, then $\widehat{\alpha}=\alpha^{(\sigma-1)^{p-2}}$ has $\ell(\widehat{\alpha})=2$ and $e(\widehat{\alpha})=0$. Hence, $\langle\widehat{\alpha}\rangle$ corresponds to a solution to the embedding problem $H_{p^{3}} \rightarrow \mathbb{Z} / p$. 


\section{Corollary 5.7.}

(1) $\nu\left(H_{p^{3}}\right)=1$;

(2) $\nu\left(A_{p} \rtimes \mathbb{Z} / p\right)=p^{2}-1$;

(3) $\nu\left(A_{i} \rtimes \mathbb{Z} / p\right)=p+1$ for $2<i<p-1$;

(4) $\nu\left(A_{i} \bullet \mathbb{Z} / p\right)=p^{2}-1$ for $2<i<p-1$.

Remark. Although it is already known, we reprove statement (1) for two reasons: to again showcase a module-theoretic perspective and because statement (1) provides the necessary example in proving statements (2)-(4).

Proof. Statement (1) is a consequence of Example 5.6. In that example, we are told that there is a $\mathbb{Z} / p$-extension $K / F$ for which the embedding problem $H_{p^{3}} \rightarrow \mathbb{Z} / p$ is solvable; call this the $H_{p^{3-}}$ extension $L / F$. Combined with the fact that $\operatorname{ker}|J(F) \rightarrow J(K)|=p$, one can use this to show that $J(K) \simeq X \oplus \mathbb{F}_{p}\left[G_{1}\right]$, with $\operatorname{dim}_{\mathbb{E}_{p}}(X)=1$. But note that, in fact, $L / F$ is a solution to the embedding problem $H_{p^{3}} \rightarrow \mathbb{Z} / p$ for any $\mathbb{Z} / p$-extension $\widetilde{K} / F$, from which we can deduce that $J(\widetilde{K}) \simeq J(K)$. Hence, over each $\mathbb{Z} / p$-extension of $F$, there is a unique module isomorphic to $A_{2}$ and generated by an element of trivial index. Each of these modules corresponds to the same $H_{p^{3}}$ extension $L / F$.

One can also prove this result by thinking of $H_{p^{3}}$ as an extension of $\mathbb{Z} / p \times \mathbb{Z} / p$ by $\mathbb{Z} / p$. Consider the field $F$ from Example 5.6 again, and note that $F$ has a unique $\mathbb{Z} / p \times \mathbb{Z} / p$ extension $K$. Because any $H_{p^{3}}$-extension of $F$ contains a unique $\mathbb{Z} / p \times \mathbb{Z} / p$ quotient extension over $F$, we see that any $H_{p^{3}}$ extension of $F$ contains $K / F$.

Now recall that [21, Theorem 6.6.1] tells us that, if $K=F(\sqrt[p]{a}, \sqrt[p]{b})$, and if $w \in F(\sqrt[p]{a})$ is an element so that $L=F(\sqrt[p]{w}, \sqrt[p]{b})$ is an $H_{p^{3}}$ extension of $F$, then all other solutions to the $H_{p^{3}}$ embedding problem over $K / F$ take the form:

$$
L_{f}:=K(\sqrt[p]{f w}, \sqrt[p]{b}) .
$$

But notice that, since $K / F$ is the unique $\mathbb{Z} / p \times \mathbb{Z} / p$ extension of $F$, we have $\sqrt[p]{f} \in K$; it follows that $L_{f}=L$, and so $F$ has a unique $H_{p^{3}}$ extension. 
The proofs of Corollary $5.7(2)-(4)$ are all relatively similar and use Example 5.6 to establish upper bounds; we will prove statement (2) and leave the verification of the other two statements to the reader. So, let $F$ be the field from Example 5.6, and note that, for each $\mathbb{Z} / p$-extension $K / F$, there are $p$ modules isomorphic to $A_{p}$. Each of these corresponds to a distinct solution to the embedding problem $A_{p} \rtimes G_{1} \rightarrow G_{1}$. By Lemma 5.3, the solutions to this embedding problem over each of the $\mathbb{Z} / p$-extensions of $F$ are distinct. Hence, we have $\nu\left(A_{p} \rtimes G_{1}\right) \leq p^{2}+p$.

For the lower bound, observe that, if there is a single $A_{p} \rtimes G_{1^{-}}$extension of a field $F$, then the $\mathbb{Z} / p$-subextension $K / F$ corresponding to the natural projection $A_{p} \rtimes G_{1} \rightarrow G_{1}$ has the property that $J(K)$ contains a module $\langle\gamma\rangle$ isomorphic to $A_{p}$. If we choose an element $\chi$ as in Proposition 3.6, then each of the modules $\langle\gamma+c \chi\rangle$ for $c \in\{0,1, \ldots$, $p-1\}$ are also isomorphic to $A_{p}$. Observe additionally that any of the other $p \mathbb{Z} / p$-extensions of $F$ admit a solution to the embedding problem $A_{2} \rtimes G_{1}$ (i.e., the $H_{p^{3}}$ subextension from the original $A_{p} \rtimes G_{1}$ extension), and hence by Proposition 4.1 (1), also admits at least one solution to the embedding problem $A_{p} \rtimes G_{1} \rightarrow G_{1}$. Since we have already argued that one such solution forces the appearance of $p$ solutions, this tells us that $F$ must have at least $p^{2}+p$ extensions with Galois group $A_{p} \rtimes G_{1}$.

Acknowledgments. We gratefully acknowledge discussions and collaborations with our friends and colleagues, I. Efrat, J. Gärtner, S. Gille, D. Hoffmann, J. Labute, J. Swallow, N.D. Tan, A. Topaz, R. Vakil and K. Wickelgren, which influenced our thinking and the results presented in this paper. We are also grateful to the referee for his/her valuable suggestions which we used to improve the exposition of this paper.

\section{REFERENCES}

1. F. Anderson and K. Fuller, Rings and categories of modules, Grad. Texts Math. 13, Springer-Verlag, New York, 1973.

2. F. Bertrandias and J.J. Payan, Г-extensions et invariants cyclotomiques, Ann. Sci. Ecole. Norm. 5 (1972), 517-543.

3. F. Bogomolov and Y. Tschinkel, Universal spaces for unramified Galois cohomology, in Brauer groups and obstruction problems: Moduli spaces and arithmetic, A. Auel, et al., eds., 2014, available at http://www.math.nyu.edu/ tschinke/ papers/yuri/14bloch/bloch18.pdf. 
4. F. Bogomolov and Y. Tschinkel, Commuting elements in Galois groups of function fields, in Motives, polylogarithms and Hodge theory, F. Bogomolov and L. Katzarkov, eds., International Press, 2002, 75-120.

5. , Introduction to birational anabelian geometry, in Current developments in algebraic geometry, L. Caporaso, et al., eds., MSRI Publ. 59, Cambridge University Press, Cambridge, 2012.

6. G. Brattström, On p-groups as Galois groups, Math. Scand. 65 (1989), 165174 .

7. S. Chebolu, I. Efrat and J. Mináč, Quotients of absolute Galois groups which determine the entire Galois cohomology, Math. Annal. 352 (2012), 205-221.

8. D. Dummit and R. Foote, Abstract algebra, 2nd edition, Prentice Hall, Upper Saddle River, NJ, 1999.

9. I. Efrat, The Zassenhaus filtration, Massey products, and representations of profinite groups, Adv. Math. 263 (2014), 389-411.

10. , Filtrations of free groups as intersections, Arch. Math. (Basel) 103 (2014), 411-420.

11. I. Efrat and J. Mináč, On the descending central sequence of absolute Galois groups, Amer. J. Math. 133 (2011), 1503-1532.

12. Galois groups and cohomological functors, Trans. Amer. Math. Soc. (2016), http://dx.doi.org/10.1090/tran/6724, in press; available at http: //arxiv.org/abs/1103.1508.

13. Small Galois groups that encode valuations, Acta. Arith. 156 (2012), 7-17.

14. M. Fried and M. Jarden, Field arithmetic, Ergeb. Math. Grenz. 11, Springer, Berlin, 2005.

15. P. Gille and T. Szamuely, Central simple algebras and Galois cohomology, Cambr. Stud. Adv. Math. 101, Cambridge University Press, Cambridge, 2006.

16. M. Hall, The theory of groups, Macmillian Company, New York, 1959.

17. M. Hopkins and K. Wickelgren, Splitting varieties for triple Massey products, J. Pure Appl. Algebra 219 (2015), 1304-1319.

18. V.V. Ishkhanov, B.B. Luré and D.K. Faddeev, The embedding problem in Galois theory, Transl. Math. Mono. 165, American Mathematical Society, Providence, 1997.

19. C.U. Jensen, On the representations of a group as a Galois group over an arbitrary field, de Gruyter, Berlin, 1989.

20. , Finite groups as Galois groups over arbitrary fields, Part 2, Contemp. Math. 131, American Mathematical Society, Providence, 1992.

21. C.U. Jensen, A. Ledet and N. Yui, Generic polynomials: Constructive aspects of the inverse Galois problem, Math. Sci. Res. Inst. Publ. 45, Cambridge University Press, Cambridge, 2002.

22. V. Kac and P. Cheung, Quantum calculus, Springer, Berlin, 2002.

23. S. Lang, Algebra, 3rd edition, Grad. Texts Math. 211, Springer, New York, 2005 . 
24. A. Ledet, Brauer type embedding problems, Fields Inst. Mono. 21, American Mathematical Society, Providence, 2005.

25. N. Lemire, J. Mináč, A. Schultz and J. Swallow, Galois module structure of Galois cohomology for embeddable cyclic extensions of degree $p^{n}$, J. Lond. Math. Soc. 81 (2010), 525-543.

26. R. Massy, Construction de p-extensions Galoisiennes d'un corps de caractéristique différente de $p$, J. Algebra 109 (1987), 508-535.

27. I. Michailov, Induced orthogonal representations of Galois groups, J. Algebra 322 (2009), 3713-3732.

28. , Four non-abelian groups of order $p^{4}$ as Galois groups, J. Algebra 307 (2007), 287-299.

29. J. Milnor, Algebraic K-theory and quadratic forms, Invent. Math. 9 (1970), $318-344$.

30. J. Mináč, M. Rogelstad and N.D. Tan, Dimensions of Zassenhaus filtration subquotients of some pro-p-groups, Israel J. Math. 212 (2016), 825-855.

31. J. Mináč, A. Schultz and J. Swallow, Galois module structure of the pthpower classes of cyclic extensions of degree $p^{n}$, Proc. Lond. Math. Soc. 92 (2006), 307-341.

32. - Automatic realizations of Galois groups with cyclic quotient of order $p^{n}$, J. Th. Nombr. Bordeaux 20 (2008), 419-430.

33. J. Mináč and M. Spira, Witt rings and Galois groups, Ann. Math. 144 (1996), 36-60.

34. , Formally real fields, Pythagorean fields, $C$-fields and $W$-groups, Math. Z. 205 (1990), 519-530.

35. J. Mináč and J. Swallow, Galois embedding problems with cyclic quotient of order $p$, Israel J. Math. 145 (2005), 93-112.

36. J. Mináč and N.D. Tan, Triple Massey products over global fields, Doc. Math. 20 (2015), 1467-1480.

37. Triple Massey products and Galois theory, J. European Math. Soc. (2016), in press.

38. The kernel unipotent conjecture and the vanishing of Massey products of odd rigid fields, Adv. Math. 273 (2015), 242-270.

39. J. Neukirch, A. Schmidt and K. Wingberg, Cohomology of number fields, 2nd edition, Springer-Verlag, Berlin, 2008.

40. A. Pfister, Eine Bemerkung zum Normenresthomomorphismus $h: K^{*} F / 2$ $\rightarrow H^{*}(F, \mathbb{Z} / 2)$, Arch. Math. 81 (2003), 272-284.

41. - On the Milnor conjectures: History, influence, applications, Jber. Math-Verein. 102 (2000), 15-41.

42. R.C. Pierce, Associative algebras, Grad. Texts Math. 88, Springer-Verlag, New York, 1982.

43. F. Pop, On Grothendieck's conjecture of birational anabelian geometry, Ann. Math. 139 (1994), 145-182. 
44. F. Pop, On the birational program initiated by Bogomolov I, Invent. Math. 187 (2012), 511-533.

45. A. Schultz, Parameterizing solutions to any Galois embedding problem over $\mathbb{Z} / p^{n} \mathbb{Z}$ with elementary p-abelian kernel, J. Algebra 411 (2014), 50-91.

46. I.R. Shafarevich, Construction of fields of algebraic number fields with given solvable groups, Izv. Akad. Nauk 18 (1954), 525-578; Amer. Math. Soc. Transl. 4 (1956), 185-237 (in English).

47. I.R. Shafarevich, Factors of a decreasing central series, Math. Z. 45 (1989), 114-117; Math. Notes 45 (1989), 262-264 (in English).

48. , On the construction of fields with a given Galois group of order $\ell^{\alpha}$, Uzv. Akad. Nauk 18 (1954), 261-296; Amer. Math. Soc. Transl. 4 (1956), 107-142.

49. V. Srinivas, Algebraic K-theory, reprint of 2nd ed., in Modern Birkhäuser classics, Birkhauser, Boston, 2008.

50. A. Topaz, Abelian-by-central Galois groups of fields I: A formal description, Trans. Amer. Math. Soc., to appear.

51. Commuting-liftable subgroups of Galois groups II, J. reine angew. Math., to appear.

52. V. Voevodsky, On motivic cohomology with Z/l-coefficients, 174 (2011), 401-438.

53. W. Waterhouse, The normal closures of certain Kummer extensions, Canad. Math. Bull. 37 (1994), 133-139.

54. G. Whaples, Algebraic extensions of arbitrary fields, Duke Math. J. 24 (1957), 201-204.

55. E. Witt, Konstruktion von galoisschen Körpern der Charakteristik $p$ zu vorgegebener Gruppe der Ordnung p ${ }^{f}$, J. reine angew. Math. 174 (1936), 237-245.

Department of Mathematics, Illinois State University, Campus Box 4520, NORMAL, IL 61790

Email address: schebol@ilstu.edu

Department of Mathematics, Middlesex College, University of Western Ontario, London, Ontario N6A5B7 Canada

Email address: minac@uwo.ca

Department of Mathematics, Wellesley College, 106 Central Street, WELLESLEy, MA 01702

Email address: andrew.c.schultz@gmail.com 